\title{
Insights on the role of forest cover and on the changes in forest cover on thirty-five endangered mammal species distributions
}

$1_{\text {Polytechnic Univer- }}$ sity of Tirana, Faculty of Civil Engineering, Department of Environmental Engineering, $R r$

M. Gjollesha, No. 4, 1023 Tirana, Albania.

Corresponding author E-mail:kuendalaze@ gmail.com

Kuenda Laze $1^{*}$

\begin{abstract}
The changes in forest cover can determine the survival of terrestrial endangered mammal species in the wild. This study assessed the impacts of forest cover changes on endangered mammal species distribution at global scale aiming to understand how the changes in forest cover may have impacted the distributions of 35 endangered small and large-body terrestrial mammals. There were used forest data obtained from time-series analyses of Landsat images between 2000 and 2014, species occurrence records collected by observations between 2000 and 2015 of Global Biodiversity Information Facility and species range data of International Union for Nature Conservation (IUCN) of the year 2015, to test the 'natural and resource conditions' hypothesis. Hypothesis on 'natural and resource conditions' produced models with high prediction accuracy of above 70 percent for 88 percent of 35 species models. The changes in forest cover explained species occurrences in 10 percent of all species models. In average, 59 percent of species occurrence records overlapped with species range data. The 51 percent of all species had no occurrence records between 2000 and 2015. Species and forest data collection as well as transnational cooperation for conservation of species roaming in the wild in upland forested areas and in cross-border areas may be critical for endangered mammal species conservation.
\end{abstract}

\section{KEYWORDS}

Endangered mammal species, forest cover, forest data, species distributions, species occurrences, threshold.

(c) BY-NC-ND $\odot 2019$ Kuenda Laze

This is an open access article distributed under the Creative Commons Attribution-NonCommercial-NoDerivs license

\section{INTRODUCTION}

The endangered terrestrial mammal species inhabit forests. All endangered mammal species are important to nature and human. The endangered mammal species are protected by national laws and international conventions aiming to species conservation. Yet, endangered mammals are at a higher risk of extinction compared to the non-protected species from industrial-scale forest conversion for agricultural plantations, heading towards the least disturbed, remote and most preferable forests for endangered mammal species (e.g., oil palm plantations are threatening great apes (Wich et al., 2014) and forest-dwelling wildlife (Strindberg et al., 2018).

Historically, human has changed the mammal diversity patterns (Faurby \& Svenning, 2015). Long-term temporal human activities on wildlife and plant diversity can affect their distribution in space leading to a potential increase of threat- ened plant species (Feng et al., 2017), of animal species like large-body carnivore, for example, Giant otter (Antunes et al., 2016), as well as to a megafauna loss (Barnosky et al., 2016).

Deforestation is one of the prime causes of global biodiversity decline due to species habitat loss (Pimm \& Raven, 2000). Expansion of agriculture land and pastureland has led croplands and pastures to become one of the largest terrestrial biomes contributing to forests' biodiversity loss (Foley et al., 2005). IUCN (2016) confirms that species habitat loss and species utilisation are the key threats of the threatened species that have a high risk of extinction in the wild. Globally, twentytwo percent of all mammal species were threatened (critically endangered, endangered and vulnerable) with habitat loss and species utilization as major threats, pastureland and cropland as possible threats, while forests, shrub lands and grasslands as the most common preferable habitats by terrestrial mam- 
mal species (IUCN 2016). Endangered species are placed between critically endangered and vulnerable species, and are considered 'to be facing a very high risk of extinction in the wild' (IUCN 2012).

Species distribution modelling (SDMs) are used to produce spatially explicit predictions in relation to the relationships between a species and its environment (Elith \& Leathwick, 2009) using a larger and a more complex data collected for species as well as environmental data from remote sensing. The most comprehensive data for the threatened species is provided by International Union for Nature Conservation (IUCN). These threatened species data were used in calculating the range map commission error (Di Marco et al., 2017), species-area relationships (Kehoe et al., 2016) and species distribution of mammals at global level (Ceballos \& Ehrlich, 2006). These species data could also be useful to quantify species and environment relationships (Johnson et al., 2004).

To effectively conserve the endangered species, there is a need to have intensive and adequate estimates on the endangered mammal species distributions and on the changes in forest cover to potentially identify any change in forests that is seriously threatening endangered mammal species by adequately considering a forest cover threshold for endangered mammal species to survive and roam in the wild. This study was undertaken to assess the impacts of forest cover changes on endangered small and large-body mammal occurrences at global scale by using species range data and forest data. This study estimated the probability of occurrences in respect to the changes in forest cover (rainforest, savanna, sub-tropical forests), land use (cropland, pastureland), and environmental conditions (elevation, slope, aspect) for thirty-five endangered mammals species. Endangered mammal species distribution models were developed based on the 'natural conditions and resources' hypothesis that defined the natural factors as driving factors for species distribution. This study did not study the historical endangered mammal species distribution, spatial scale where the animals perceived their environment and anthropological factors (e.g., human population) that limit endangered mammal species distributions. Here, the aim of the study was: 1- how the changes in forest cover from 2000 to 2014 (Hansen et al., 2013) ccould have impacted the occurrences of 35 endangered mammal species (Colby Loucks, WWF, IUCN, Maryke Gray, Alejandro Rodríguez/Cat SG, NatureServe, Panthera) as categorized by International Union for Nature Conservation (IUCN), in the year 2015. I identified the significance of forest cover (and its changes) on the probability of species occurrences for thirty-five endangered mammal species using geographic species ranges (henceforth 'species habitat areas') for eighteen endangered carnivore species and seventeen endangered noncarnivore small mammals (small-body and or semi-aquatic species) in Africa, Asia, Europe, North and South America (IUCN 2016).

\section{MATERIALS AND METHODS}

\subsection{Endangered species data}

Geographical range maps were projected from WGS 84 to UTM (Universal Transverse Mercator) coordinate system in ArcGIS 10.3 to accurately measure the Euclidean distance to the protected areas in meters, endangered mammal species habitat areas in $\mathrm{km}^{2}$ and to make all calculations for explanatory variables (see 1.2 Forest and environmental data). Using a UTM coordinate system, distortion of large habitat areas (e.g., Lycaon in Figure 1) in a continent was avoided (see e.g., Baselga et al., 2012).

Thirty-five terrestrial and semi-aquatic endangered mammal species, which preferably inhabited the forested areas according to IUCN (2016), were studied. In total, 60 percent of thirty-five endangered mammal species had a forest cover of above 30 percent. The forest cover of (above) 30 percent was an indicator of high biodiversity amongst (small) mammals (see, e.g., Estavillo et al., 2013). Cuon alpinus, Enhydra lutris Cynogale benetti, Lutra sumatrana, Tupaia nicobarica, Tupaia chrysogaster were excluded from the analysis. Cynogale benetti, Lutra sumatrana, Tupaia nicobarica, Tupaia chrysogaster were excluded from the analysis because their species habitat areas covered entire island areas ( 1 couldn't fulfil the criterion of pseudo-absence selection; see 1.3 Endangered species models). Cuon alpinus had a very large and complex range data to finalize the analysis. Enhydra lutris was an aquatic species, and its habitat area was disconnected from the forests. Mustela nigripes was a reintroduced species and it was included in this analysis (Mustela in Figure 1). The thirty-five species are distributed in Europe, Asia, Africa, South America and North America (black and grey colour dots), Figure 1.

Biodiversity Assessment Unit, which is a part of the IUCN Species programme, assessed the data for endangered mammal species. Biodiversity Assessment Unit was established to map the distribution and assess the conservation status for each species and then to establish a global baseline for biodiversity monitoring (IUCN 2017b). IUCN species experts calculated the species habitat areas indicating spaces where a species was extant (not extinct), or where a species was found permanently or periodically (IUCN 2016). The details on endangered mammal species data, forest cover and endangered mammal species habitat area are in S1, S2 and Table S3.

I used 836 endangered mammal species occurrence records between 2000 and 2015 (GBIF.org 2018a), 986 endangered mammal species occurrence records between 2000 and 2017 (GBIF.org 2018b) based on observations, human observations and machine observations of GBIF and species habitat areas data for thirty-five endangered mammals habiting in forested continental areas (not in forested islands), (Table S3). For every endangered mammal species, I calculated the number and the percentage of species occurrence records between 2000 and 2015 (of Global Biodiversity Information Facility (GBIF)) that were found inside of endangered mammal species habitat areas. 


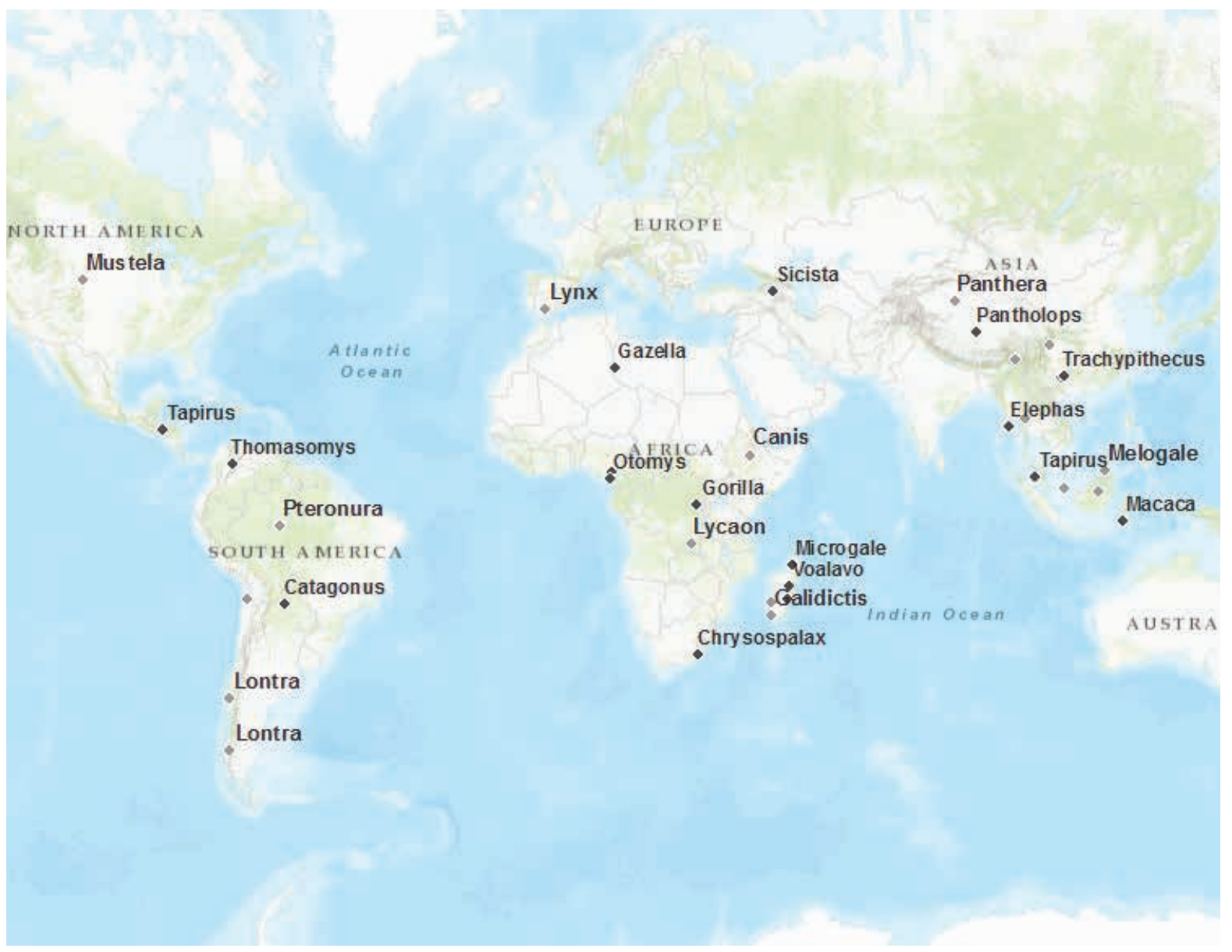

Figure 1: Endangered mammal species habitat locations.

I used endangered mammal species habitat areas (of IUCN) to randomly select a priory 30 species occurrences (points) for every endangered mammal species (Figure 1) employing Create Random Points in ArcGIS 10.3., arguing that every endangered mammal species would be present within its endangered mammal species habitat areas and would be absent outside of its endangered mammal species habitat areas. For every endangered mammal species, I created a mask (polygon feature class in ArcGIS 10.3 with a larger area than an endangered mammal species habitat area) to include at least one protected area point data (IUCN and UNEP-WCMC 2015) outside the endangered mammal species habitat area(s) for calculating the distance to the nearest protected area variable and to randomly select thirty pseudo-absences point data (also located outside endangered mammal species habitat area(s)). The selection of pseudo-absences followed three conditions as follows: (1) the number of pseudo-absences was equal to the number of endangered mammal species occurrences records for every endangered mammal species, (2) only one pseudoabsence location (point) was selected in a range of at least 1 km far from any other pseudo-absence locations, (3) pseudoabsences were preferably located in the forest areas outside (endangered mammal) species habitat areas because generating pseudo-absences further away from the optimum estab- lished by the occurrence record endangered mammal species data may increase over-prediction of the model (Chefaoui \& Lobo, 2008).

\subsection{Forest and environmental data selection}

Forest cover change is a sum of forest cover gain (positive sign) and forest cover loss (negative sign). Changes in forest cover (obtained from LANDSAT) were caused by fires, selective logging, clearing of short cycle plantation in sub-tropical and tropical eco-zones, and rotation agricultural clearing of smallholder in dry and humid tropical forests (Hansen et al., 2013). With 'trees defined as vegetation taller than $5 \mathrm{~m}$ height', forest cover in year 2000 , forest cover loss, forest cover gain was calculated in percentage per output grid cell of approximately $900 \mathrm{~m}^{2}$ (Hansen et al., 2013). Forest cover loss was a change from a forest to non-forest state, during the period 2000-2014, and forest cover gain is a change from non-forest to forest change within the period 2000-2012 (Hansen et al., 2013). I used forest cover data (approximately $900 \mathrm{~m}^{2}$ ) of Hansen et al. (2013) for the year 2000 .

Terrestrial Protected Areas (point) data of the version July 2015 of the World Database on Protected areas (IUCN and UNEP-WCMC 2015) were used. The minimum Euclidean distances of species occurrences and pseudo-occurrence point 
data (see 1.3. Endangered species models) to the nearest protected areas (point data) were calculated by applying straight line in Spatial Analyst in ArcGIS 10.3 (Environmental Systems Research Institute 2016) producing a raster.

I used the total cultivated land, grass, scrub and woodland and forest cover grid data presenting the percentage share of the total grid-cell (of 5 minute latitude by 5 minute longitude grid cell) of Food and Agriculture Organisation (FAO) for the year 2000 (Fischer et al., 2008), and global and continental pastureland and cropland raster grid data $(5 \mathrm{~km} \times 5 \mathrm{~km})$ that ranged from 0 to 1 (Ramankutty et al. 2010). The zero and one was respectively 0 percent and 100 percent of pixel area under either pastureland or cropland. Elevation raster data (1-minute arc) were respectively retrieved from the website of IUCN (IUCN 2017). Slope and aspect raster data (1-minute arc) were derived from the elevation data using Slope and Aspect in Spatial Analyst in ArcGIS 10.3, respectively. Terrain Ruggedness Index, diversity and fragmentation of forest patches (using forest cover of the year 2000) were respectively calculated using Morphometry and Pattern Analysis of SAGA GIS 6.1.0 (Conrad et al., 2015). A value of a raster (elevation, slope, aspect, distance to the nearest protected areas, pastureland, cropland, forest cover and forest cover change) was extracted and assigned to every (endangered mammal species) occurrence and pseudo-occurrence points. All variables were calculated using the extensions available in ArcGIS 10.3 (Environmental Systems Research Institute 2016) and SAGA GIS 6.1.0 (Conrad et al., 2015). All information on variables is in Table S4.

\subsection{Endangered species models}

I identified an a priory hypothesis on groups of variables that drive the occurrences of 35 endangered mammals species based on environmental conditions and resources that are required for species reproduction and survival (Table 1). I developed species 'natural conditions and resources' model by following an approach of Naves et al. (2003) that was developed for brown bear in Spain. 'Natural conditions and resources' models included environmental variables (elevation, slope and aspect, Terrain Ruggedness Index), forest cover, forest patches diversity, forest patches fragmentation, cropland and pastureland. Forest cover and forest cover changes were related to food abundance of the species (Table 1). The 'natural conditions and resources' hypothesis is in $\mathrm{S} 5$.

For every endangered mammal species, a priory of thirty occurrences within endangered mammal species habitat areas data and thirty pseudo-absences of species outside of endangered mammal species habitat areas were randomly selected using ArcGIS 10.3. The same number of occurrences and pseudo-absences was assumed for all endangered mammal species ignoring species type information (large-body mammal, small-body mammal, carnivore, non-carnivore, terrestrial, semi-aquatic mammal), species reproduction rate, endangered species population number, and range map commission (Di Marco et al. 2017) in modelling.

Preceding regression analysis, I firstly removed the most highly correlated variables (Pearson correlation test; $r>$ $0.7)$ and less correlated variables were tested for their significant differences with occurrence and pseudo-absence locations of the mammal species (Kruskal-Wallis test; $p>0.05$ ). Spatial autocorrelation (SAC) could be present in the dependent variable (species occurrences and pseudo-absences) because regions (areas) of an identified species occurrence could be sampled with higher intensity than other areas of unidentified species occurrences (e.g., Red List Species) (Dormann, 2007). The spatial autocorrelation of the dependent variables, for every species, was calculated using the software Geoda 1.8.12 (Anselin et al., 2006) for the spatial dependence of data (Anselin 1988) (similarity between species occurrences data).

Generalized linear models (GLM) with logit-link was used to relate the occurrence and pseudo-absence data to explanatory variables to predict the probability of a given endangered mammal species. GLMs are an extension of classic linear regression models (McCullagh \& Nelder 1989). Binomial error structure (logistic regression) was used for these endangered mammal species data. A logistic regression model predicts the probability of a given endangered mammal species occurrence (estimated high probability values and estimated low probability values of endangered mammal species occurrence would respectively mean suitable habitats and unsuitable habitats for a given endangered mammal species) at a given point location within the species study area. All GLMs were fitted within the program R 2.11.0 (R Development Core Team 2011) using the

Table 1: Hypothesis of 'Natural conditions and resources' based on biotic and abiotic factors acknowledged for shelter, food and reproduction of endangered species mammals in the wild

\begin{tabular}{|c|c|c|}
\hline Hypothesis & Description & References \\
\hline $\begin{array}{l}\text { Natural conditions } \\
\text { and resources }\end{array}$ & $\begin{array}{l}\text { The endangered mammal species need forests, high elevation to hide, breed } \\
\text { and search for food. Lynx pardinus and Panthera tigris need stable and undi- } \\
\text { sturbed dense forests elevated land and topography for food and refuge and } \\
\text { breed at landscape level. Primates would mostly occupy areas with a tree } \\
\text { (forest) cover above } 50 \text { percent and small mammals above } 30 \text { percent. Small } \\
\text { mammals tend to use forests, surrounded by pastures and tree crops and } \\
\text { can use farmland for food search. Threatened terrestrial mammal species } \\
\text { can possibly occupy frontier landscape for refuge (shelter). }\end{array}$ & $\begin{array}{l}\text { (Estavillo et al., 2013; Fernández et al., } \\
\text { 2003; Kanagaraj et al., 2011; Rogers \& } \\
\text { Gorman, 1995; Fernandez et al., 2006; } \\
\text { Nüchel et al., 2018; Ester et al., 2018) }\end{array}$ \\
\hline
\end{tabular}


packages of 'bbmle', 'biodiversityR', 'biomod2', 'brglm', 'DAAG', 'foreign', 'glmmboot', 'verification'.

There were thirty-five candidate models fitted (one GLM model for each species). Model performance was evaluated with Akaike Information Criterion (AIC) and adjusted- $\mathrm{R}^{2}$ for GLM models. Regression analysis was repeated for every endangered mammal species (thirty-five) to calculate the coefficients in terms of their sign and magnitude and significance ( $p$-value $<5$ percent). The estimated probability of species occurrence were from 0.5 (e.g., Naves et al., 2003) to 1.00 to set a threshold (average value $H$ in Figure 3), (e.g., Liu et al., 2005) for separating occurrences with high probability values (suitable habitat) and with low probability values (marginal and non-suitable habitat). The results of the selected model were mapped out to compare the prediction of endangered mammal species occurrences with endangered mammal species habitat area. Areas with probability values above the threshold were identified as a potential species conservation area.

\subsection{Model evaluation}

The endangered mammal species models were evaluated using two approaches. All models were evaluated for discrimination success by calculating the area under a receiver operating characteristic curve (AUC). The value of the AUC ranged from zero to one. $A \cup C=0.5$ indicated a random prediction, $A U C<$ 0.7 was considered poor and AUC $>0.8$ indicated high model ability to discriminate between high estimated probability of species occurrence (e.g., above threshold of 0.50 ) and low estimated probability of species occurrence (e.g., below threshold of 0.50) (Hosmer \& Lemeshow, 2000). In addition, the species models were evaluated using the true skill statistic (TSS), which is an alternative measure of model accuracy where kappa is independent of prevalence (Allouche et al., 2006). TSS has values between 0 and 1 . TSS values between 0.5 and 0.7 indicate a good model prediction, between 0.7 to 0.85 a very good prediction and between 0.85-0.9 an excellent prediction (Nüchel et al., 2018). I also ran permutation test for GLM estimated coefficients (10000 times) for a higher accuracy (Hesterberg, 2015) and Confidential Interval (Cl) with alpha level of 0.10 ('glmmboot' package of R Development Core Team (2018)).

First, I calculated the predictive accuracy of our endangered mammal species models using the deviance explained in percentage $\left(D^{2}\right)$ and a cross-validation approach. Cross-validation was used for logistic regression with a binary dependent variable to check if there was over-fitting in the fitted models (Fernández et al. 2003; Kanagaraj et al. 2011). I ran cross-validation. I divided our data into 10 and 100 parts (folds). Nine folds were used for fitting the model and the tenth fold was used for model testing, in the case of 10 folds (the same procedure was followed for cross-validations of 100 folds). The value of cross-validation was calculated as an average value of probability value from 0 to 1 (DAAG package of R Development Core Team (2011)). For every endangered mammal species, I ran our species models by randomly removing one, two and four occurrence and replaced with randomly selected locations (no occurrence) of species and ran the GLM model.

Second, I calculated the presence probability of occurrences based on species (GLM) models for every species using Maximum Entropy Presence (Phillips et al. 2006). I used a MaxEnt Package in C++ programming language of Dekan Lin in SAGA GIS 6.1.0. I compared the logistic regression (GLM) model predictions and presence probability of occurrence for every endangered mammal species. Then, I calculated the presence probability of occurrence records (based on the species (GLM) models) using GBIF species occurrence between 2000 and 2015 (and cultivated land and forest data of FAO). I compared the average estimated probability obtained from GLM models with the presence probability of occurrence obtained from MaxEnt. Finally, I overlapped the GBIF species occurrence records 1- between 2000 and 2015 and 2- between 1885 and 2017 (i.e., without a time filter but filtering by observations) with endangered mammal species habitat areas of IUCN to calculate the number of GBIF occurrence records and their percentage (out of total number of GBIF occurrence records) in species habitat areas, for every endangered mammal species.

\section{RESULTS}

\subsection{Model results}

Correlation between elevation and slope (Pearson's $r>=0.70$ ) was high for Macara maura endangered mammal species ( $r=0.78)$. Correlation between forest cover and the nearest distance to protected areas as well as between elevation and forest cover was respectively high for Lepilemur microdon, $(r=0.80)$ and for Tapirus indicus, $(r=1)$. For these endangered mammal species, correlated variables, for example, elevation and slope, were separated into two GLM individual multiple regression models. The GLM performances were calculated and compared in terms of estimator of AIC. The GLM model that obtained the lowest AIC value was then selected. In total, 88 percent and 94 percent of 35 GLM models respectively performed well in terms of model accuracy (the area under the Receiver Operating Characteristic Curve (AUC), AUC > 0.70 and TSS $\geq 0.50$ ), (Table 2).

Environmental variables (elevation, slope, aspect, terrain ruggedness index) were statistically significant explanatory variables ( $p$-value $<0.05$ ) for 48 percent out of 35 GLM models. Forest cover and/or diversity was respectively a statistically significant explanatory variable ( $p$-value $<0.05$ ) for 33 percent and 52 percent of estimated probability of carnivore and noncarnivore species occurrence. Forest cover and forested landscape metrics of diversity were found in 31 percent of 35 GLM models.

Forest cover change was present in two endangered mammal species models (Mungotictis decemlineata with AUC $=0.99$ and TSS $=0.83$ and Catopuma badia with AUC $=0.83$ and TSS $=0.66$ in Table 2). While forest cover contributed positively to the estimated probability of occurrence records of $\mathrm{Ca}$ - 


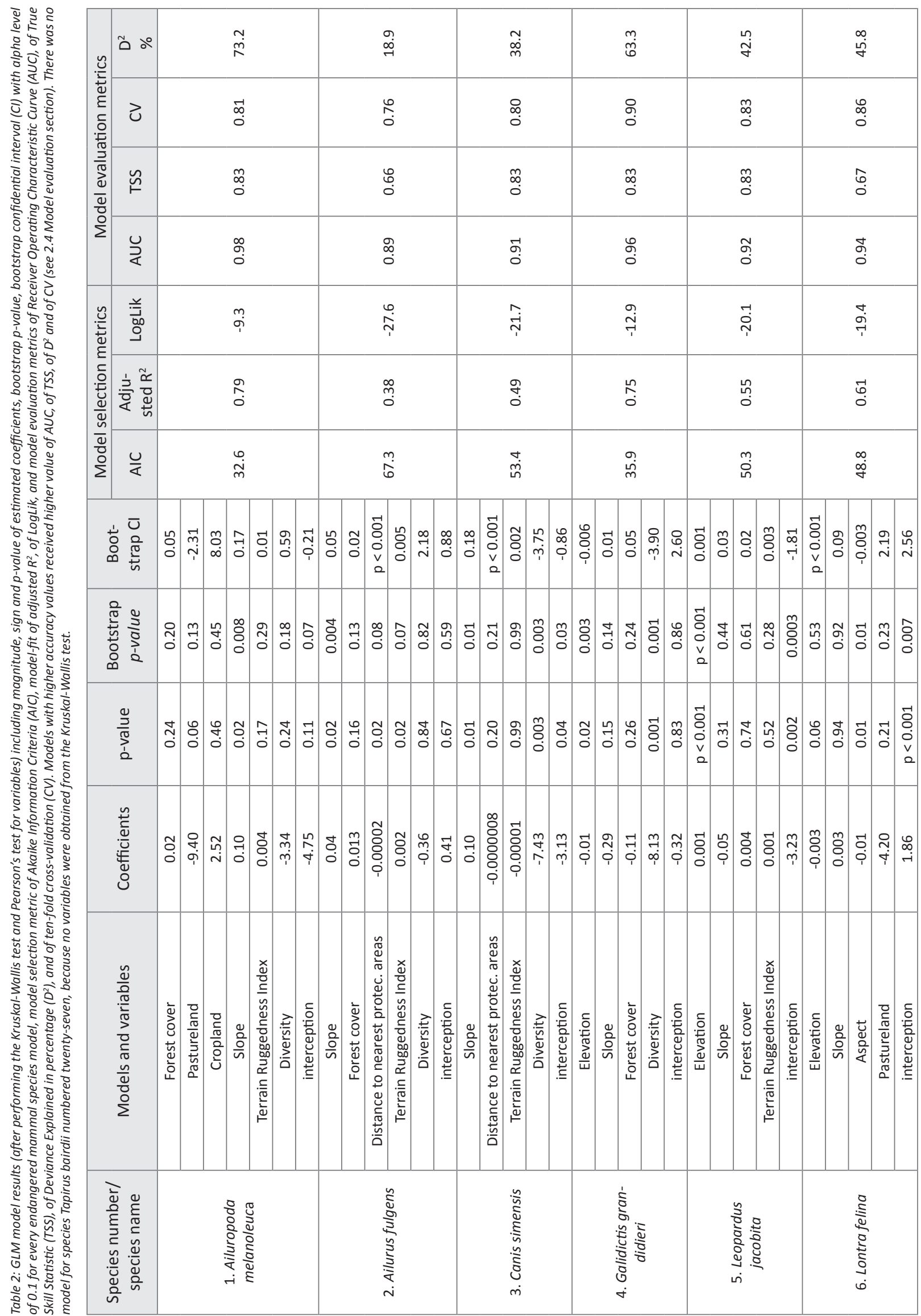




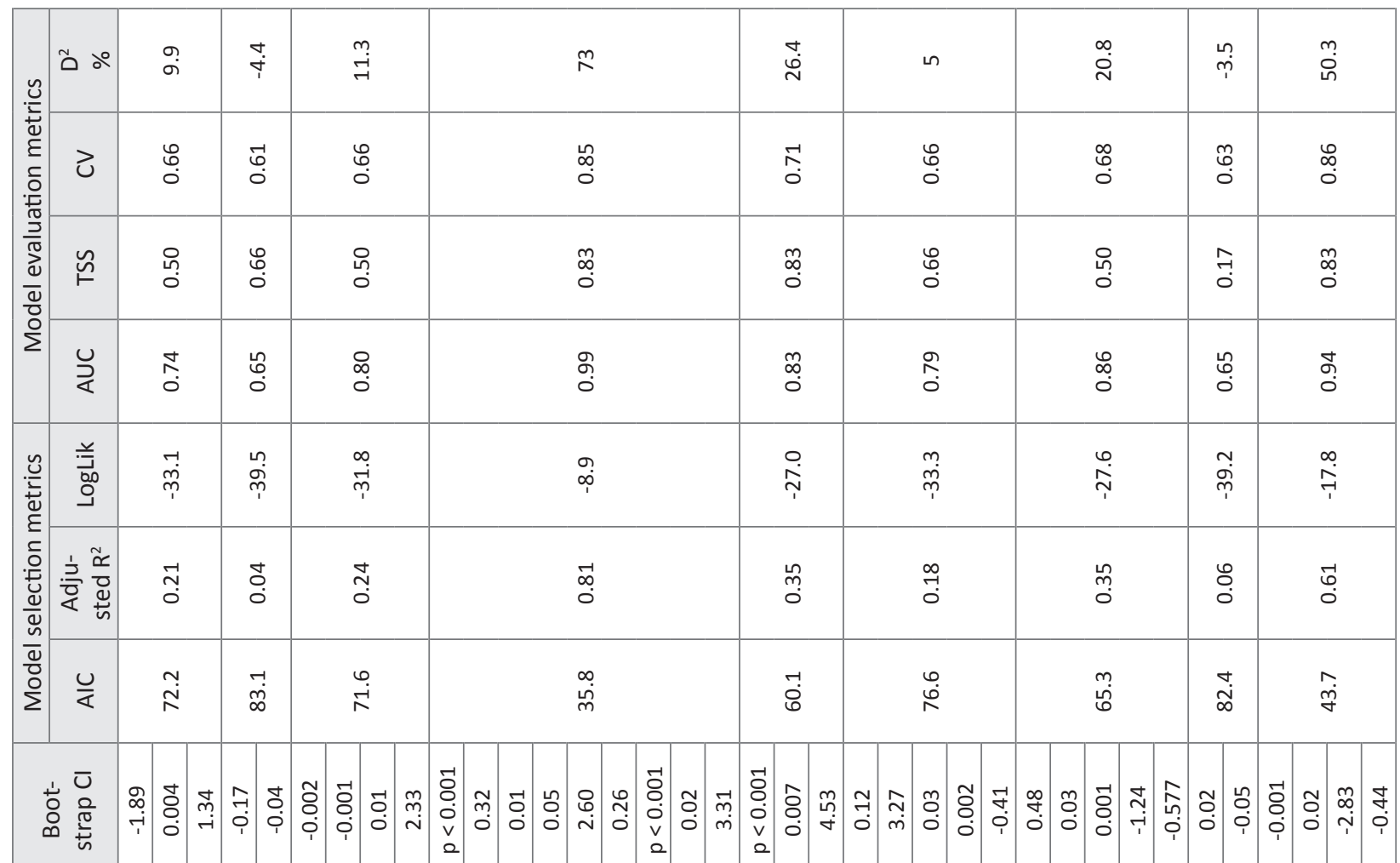

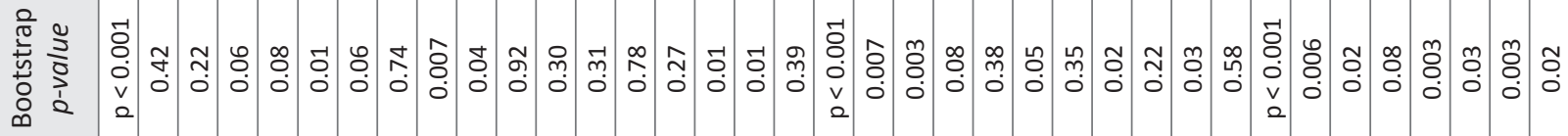

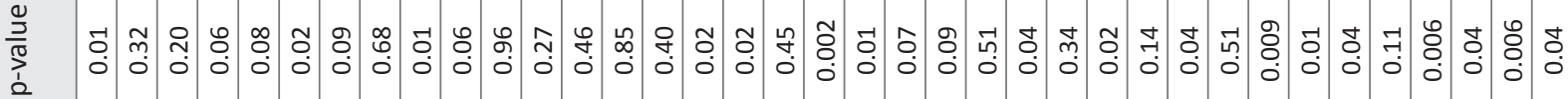

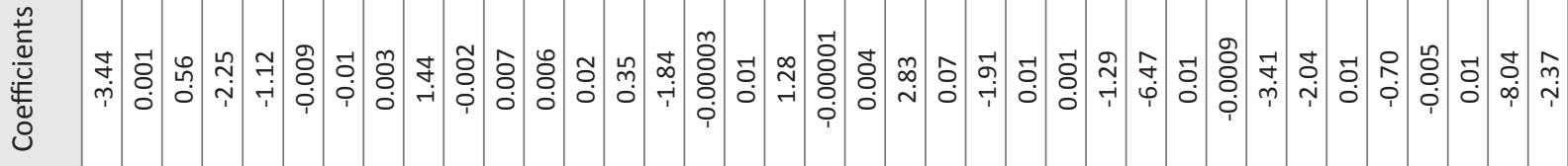

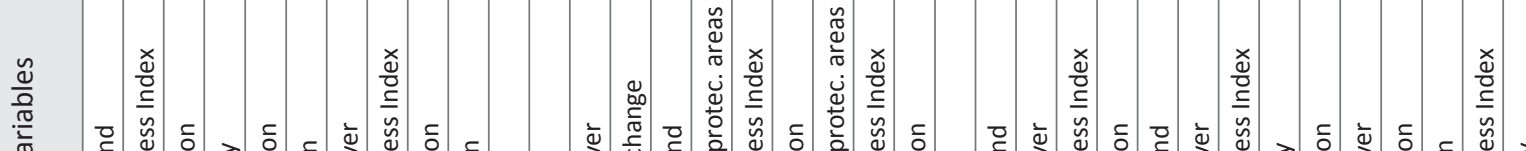

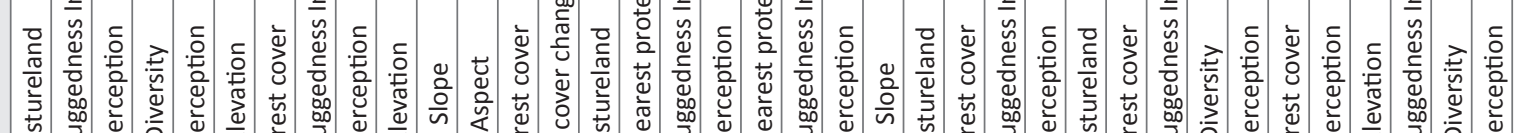

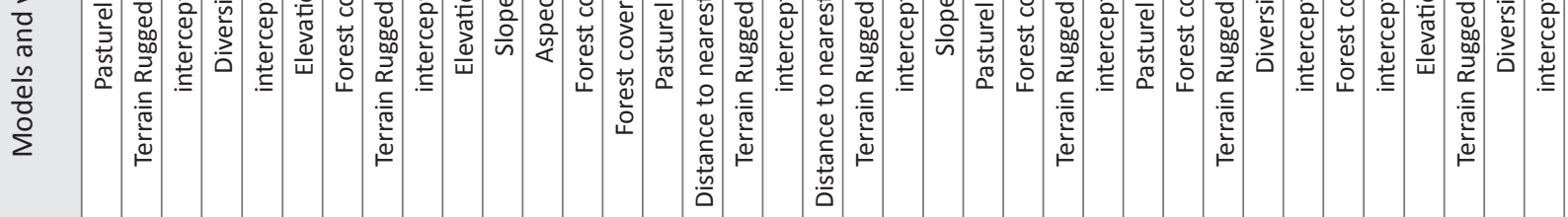

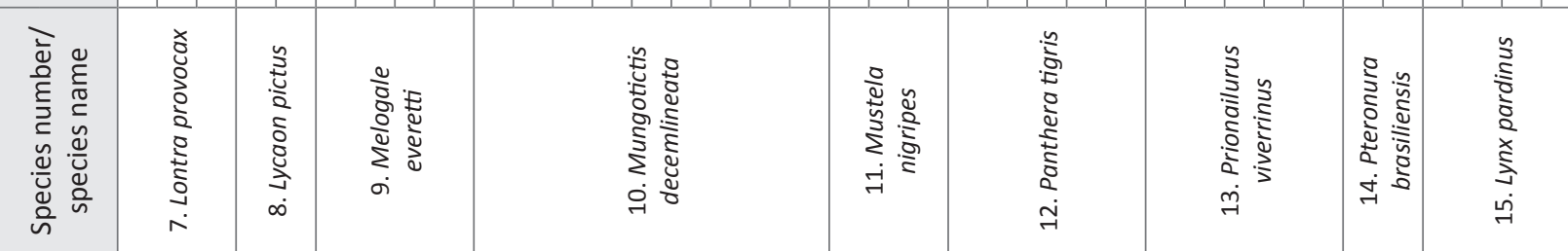




\begin{tabular}{|c|c|c|c|c|c|c|c|c|c|c|c|c|c|c|c|c|c|c|c|c|c|c|c|c|}
\hline ص & & $\begin{array}{l}\dot{\varphi} \\
\dot{\varphi}\end{array}$ & & in & & & $\frac{n}{6}$ & & & & $\stackrel{\mathscr{m}}{m}$ & & \multicolumn{5}{|c|}{ นี } & \multicolumn{3}{|c|}{ } & \multicolumn{4}{|c|}{$\begin{array}{l}\text { â } \\
\text { in }\end{array}$} \\
\hline 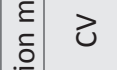 & & $\stackrel{m}{\stackrel{n}{o}}$ & & $\stackrel{\infty}{\stackrel{\infty}{0}}$ & & & $\begin{array}{l}\infty \\
\infty \\
0\end{array}$ & & & & $\stackrel{\circ}{\stackrel{0}{0}}$ & & & & $\begin{array}{l}\infty \\
\infty \\
0\end{array}$ & & & & পே. & & \multicolumn{4}{|c|}{$\stackrel{n}{\circ}$} \\
\hline$\frac{\tilde{D}}{\frac{0}{\alpha}} \mid \tilde{\curvearrowleft}$ & & $\begin{array}{l}\infty \\
\infty \\
0 \\
0\end{array}$ & & น̊ & & \multicolumn{4}{|c|}{$\stackrel{\circ}{\rightarrow}$} & \multicolumn{4}{|c|}{$\stackrel{\circ}{\rightarrow}$} & \multicolumn{4}{|c|}{$\stackrel{\substack{\infty \\
0}}{0}$} & \multicolumn{3}{|c|}{$\begin{array}{l}\mathscr{0} \\
\stackrel{0}{0}\end{array}$} & \multicolumn{4}{|c|}{$\begin{array}{c}m \\
\infty \\
0 \\
0\end{array}$} \\
\hline 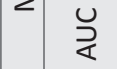 & & 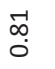 & & : & & \multicolumn{4}{|c|}{ ลิ } & & ஷุ & & & \multicolumn{4}{|c|}{$\begin{array}{l}\infty \\
\text { ơ } \\
\text { o }\end{array}$} & & ஜू & & \multicolumn{4}{|c|}{ के } \\
\hline 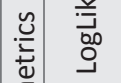 & & $\begin{array}{l}\text { o. } \\
\dot{m}\end{array}$ & & $\stackrel{m}{m}$ & & \multicolumn{4}{|c|}{ ت̃ } & & $\stackrel{\vec{p}}{i}$ & & & \multicolumn{4}{|c|}{$\stackrel{\vec{I}}{7}$} & & $\stackrel{0}{\ddot{m}}$ & & \multicolumn{4}{|c|}{ 苞 } \\
\hline 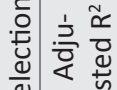 & & ָৃ. & & $\stackrel{\text { I }}{\stackrel{0}{0}}$ & & \multicolumn{4}{|c|}{$\stackrel{n}{\stackrel{n}{o}}$} & & $\stackrel{\text { qn }}{0}$ & & & \multicolumn{4}{|c|}{$\stackrel{\substack{n \\
0}}{o}$} & & $\stackrel{m}{o}$ & & \\
\hline$\frac{\bar{D}}{\Sigma} \frac{U}{\alpha}$ & & $\underset{\leftarrow}{\frac{1}{6}}$ & & $\begin{array}{l}\dot{0} \\
\stackrel{\varphi}{n}\end{array}$ & & \multicolumn{4}{|c|}{$\stackrel{n}{\dot{m}}$} & \multicolumn{3}{|c|}{ กֶ. } & \multicolumn{5}{|c|}{ 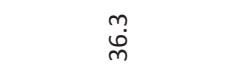 } & \multicolumn{3}{|c|}{ ت্ } & \multicolumn{4}{|c|}{$\stackrel{m}{q}$} \\
\hline 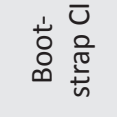 & $\begin{array}{l}\vec{b} \\
0 \\
0 \\
0 \\
0\end{array}$ & : & ?ִ & 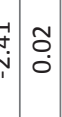 & $\stackrel{M}{\stackrel{M}{i}}$ & 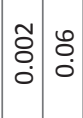 & ồ & & 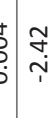 & $\hat{o}$ & 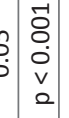 & $\underset{\sim}{\tilde{\sigma}}$ & 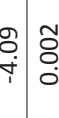 & 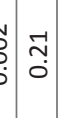 & $\begin{array}{l}0 \\
0 \\
0\end{array}$ & 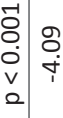 & : & 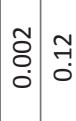 & $\mid \begin{array}{ll}\vec{b} & \alpha \\
\dot{v} & \alpha \\
v & c \\
\alpha\end{array}$ & $\begin{array}{l}y \\
0 \\
0 \\
0\end{array}$ & ò & 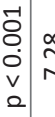 & ஸ̦ & $\begin{array}{l}n \\
\infty \\
0 \\
\dot{0}\end{array}$ \\
\hline 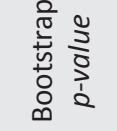 & ठ் & $\begin{array}{l}- \\
0 \\
0\end{array}$ & b. & 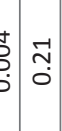 & $\stackrel{2}{\stackrel{0}{0}}$ & \begin{tabular}{|l|l|} 
& \\
0 & 0 \\
0 & 0 \\
$v$ & 0 \\
0 &
\end{tabular} & : & $\hat{m}$ & 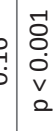 & 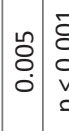 & 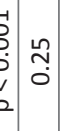 & ֻُ & \begin{tabular}{c|c}
$\vec{b}$ & \\
$\vdots$ \\
$\vdots$ \\
$\vdots$ \\
0
\end{tabular} & $\begin{array}{l}m \\
\vdots \\
0 \\
0 \\
0\end{array}$ & ㄱ: & 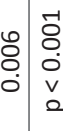 & 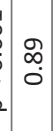 & 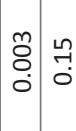 & 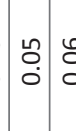 & $\dot{b}$ & શָ̣ & 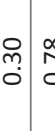 & م. & $\begin{array}{l}m \\
0 \\
0\end{array}$ \\
\hline $\begin{array}{l}\frac{0}{2} \\
\frac{2}{\pi} \\
\frac{1}{2}\end{array}$ & ষे & \begin{tabular}{l|l}
0 \\
$\vdots$ \\
$\vdots$
\end{tabular} & bis & Ș & $\stackrel{2}{2}$ & 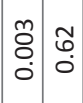 & $\begin{array}{l}\infty \\
0 \\
0 \\
0\end{array}$ & 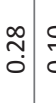 & 官 & 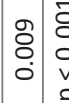 & 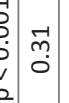 & 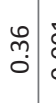 & 咅 & $\begin{array}{l}n \\
\vdots \\
\vdots \\
0 \\
0\end{array}$ & స̃ & 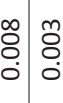 & : & $\begin{array}{ll}\tilde{m} & \vec{n} \\
0 & 0 \\
0 & 0\end{array}$ & 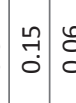 & $\stackrel{\substack{0 \\
\infty \\
\infty}}{\infty}$ & 㐌 & 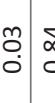 & ن. & 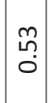 \\
\hline 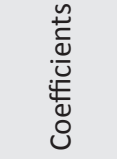 & 芩 & $\begin{array}{lll}m & 0 \\
0 & 0 & \end{array}$ & & $\begin{array}{ll}\substack{0 \\
f} \\
\dot{f}\end{array}$ & $\begin{array}{c}\tilde{m} \\
i \\
i\end{array}$ & 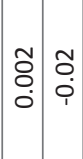 & $\begin{array}{l}0 \\
\delta \\
0\end{array}$ & 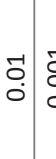 & bे & $\left.\begin{array}{l|l} \\
\hdashline \\
0\end{array}\right)$ & 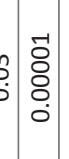 & $\stackrel{\infty}{\rightarrow} \underset{i}{\stackrel{1}{i}}$ & 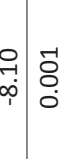 & 3 & 望 & 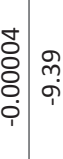 & \begin{tabular}{l}
$m$ \\
\hdashline \\
0 \\
0
\end{tabular} & 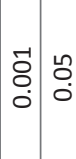 & 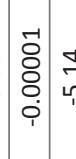 & $\hat{m}$ & $\begin{array}{l}\dot{t} \\
\varnothing \\
0\end{array}$ & 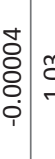 & 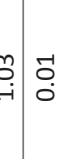 & \begin{tabular}{l}
$\infty$ \\
\hdashline \\
$i$
\end{tabular} \\
\hline $\begin{array}{l}\frac{n}{0} \\
\frac{0}{0} \\
\frac{\pi}{0} \\
\frac{0}{\pi} \\
\frac{0}{0} \\
\frac{0}{\pi} \\
\frac{n}{0} \\
\frac{0}{0} \\
\sum\end{array}$ & 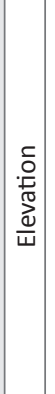 & $\begin{array}{l}0 \\
\frac{0}{0} \\
\text { ․ }\end{array}$ & 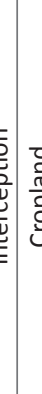 & 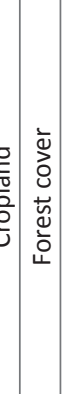 & 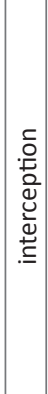 & 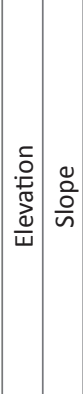 & 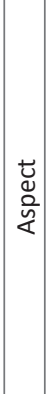 & 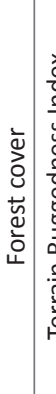 & 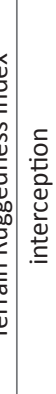 & 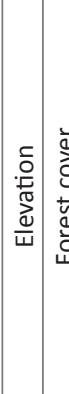 & 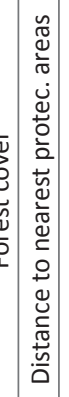 & $\begin{array}{l} \\
\frac{0}{0} \\
\frac{0}{0} \\
\frac{0}{0} \\
\stackrel{0}{0}\end{array}$ & 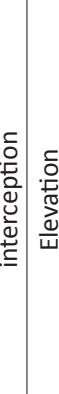 & 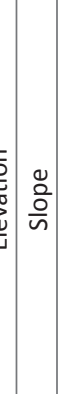 & 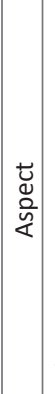 & 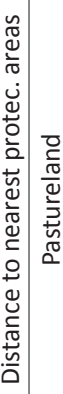 & 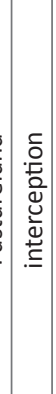 & 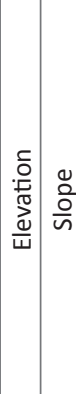 & 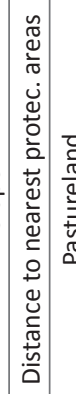 & 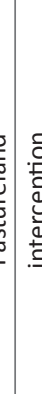 & 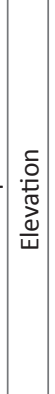 & 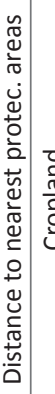 & 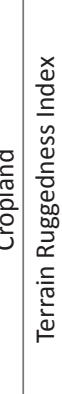 & 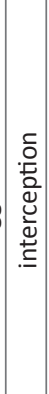 \\
\hline 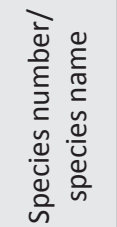 & & 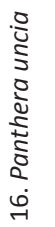 & & 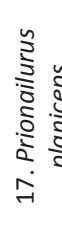 & & & 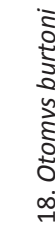 & & & & 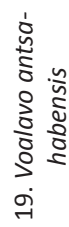 & & & & 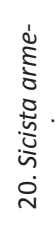 & & & & 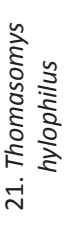 & & & & 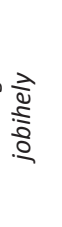 & \\
\hline
\end{tabular}




\begin{tabular}{|c|c|c|c|c|c|c|c|c|c|c|}
\hline y & ป ${ }^{\circ}$ & $\stackrel{\circ}{i}$ & ㄱ.ㅇ. & $\vec{m}$ & $\stackrel{\mathscr{N}}{\dot{d}}$ & 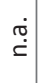 & 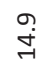 & 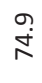 & $\begin{array}{l}\stackrel{f}{g} \\
\dot{g}\end{array}$ & $\stackrel{+}{\sim}$ \\
\hline 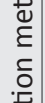 & 己 & $\stackrel{\stackrel{L}{\infty}}{\stackrel{0}{0}}$ & $\begin{array}{l}\infty \\
\infty \\
0 \\
0\end{array}$ & $\begin{array}{l}\stackrel{R}{0} \\
\text { O. }\end{array}$ & $\stackrel{\infty}{\stackrel{\infty}{0}}$ & 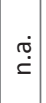 & 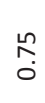 & $\stackrel{\text { ğ }}{\circ}$ & $\begin{array}{l}\infty \\
\infty \\
\infty \\
0\end{array}$ & $\stackrel{?}{\stackrel{2}{0}}$ \\
\hline $\begin{array}{l}\frac{\bar{v}}{\bar{d}} \\
\frac{d}{d}\end{array}$ & 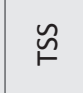 & $\begin{array}{l}\hat{b} \\
\dot{0}\end{array}$ & $\begin{array}{c}\infty \\
\infty \\
0 \\
0\end{array}$ & 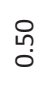 & $\begin{array}{l}\hat{0} \\
\dot{0}\end{array}$ & 苗 & ڤ్రి & 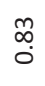 & $\begin{array}{l}m \\
\infty \\
0 \\
0\end{array}$ & $\begin{array}{c}\infty \\
\infty \\
\infty\end{array}$ \\
\hline 2 & $\stackrel{\circlearrowright}{\gtrless}$ & ๙ু & পே. & î. & 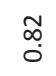 & 苗 & $\begin{array}{c}\infty \\
\infty \\
0\end{array}$ & $\begin{array}{l}\text { ğ } \\
\text { o }\end{array}$ & ñ & $\begin{array}{l}\text { o } \\
0 \\
0\end{array}$ \\
\hline 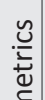 & $\begin{array}{l}\text { 兰 } \\
\text { 맘 }\end{array}$ & $\hat{\text { ஸิ }}$ & $\underset{\substack{\infty \\
\rightarrow \rightarrow}}{\rightarrow}$ & $\underset{\sim}{\stackrel{1}{~}}$ & 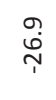 & 苗 & กั & $\underset{\phi}{\infty}$ & 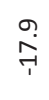 & $\stackrel{\vec{H}}{\text { ஸे }}$ \\
\hline 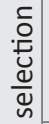 & 紊紊离 & in & $\stackrel{\overrightarrow{0}}{0}$ & $\stackrel{\stackrel{d}{S}}{0}$ & $\stackrel{\hat{m}}{o}$ & 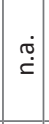 & m̊ & $\begin{array}{l}\vec{\infty} \\
0 \\
0\end{array}$ & : & $\stackrel{\vec{f}}{0}$ \\
\hline $\begin{array}{l}\frac{0}{0} \\
\frac{0}{2}\end{array}$ & $\frac{U}{4}$ & Н & $\stackrel{\stackrel{\leftrightarrow}{\dot{\gamma}}}{ }$ & $\stackrel{m}{p}$ & ભ̆ & 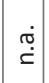 & ல் & $\stackrel{9}{\sim}$ & $\dot{g}$ & อ̃ \\
\hline
\end{tabular}

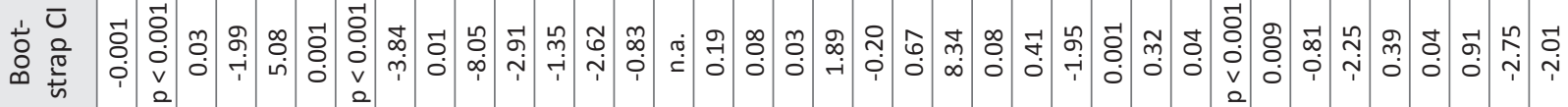

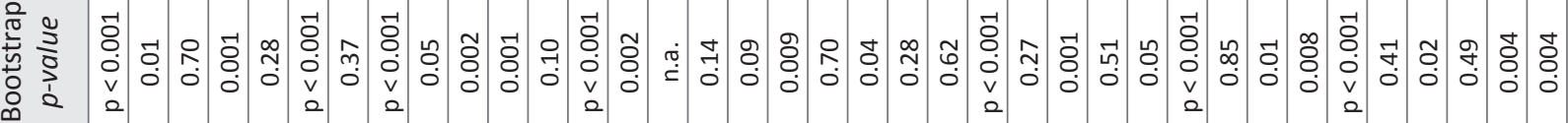

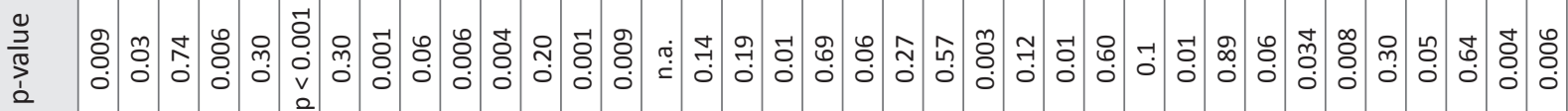

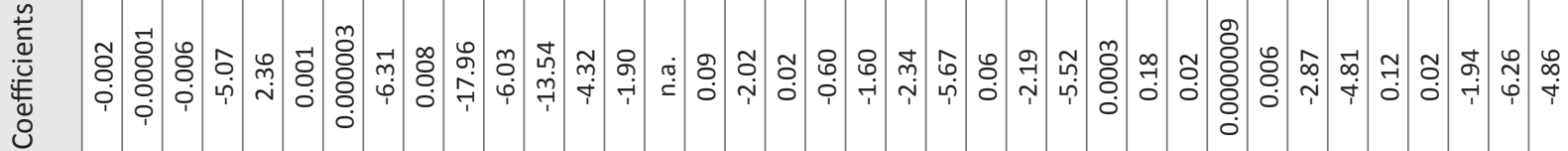

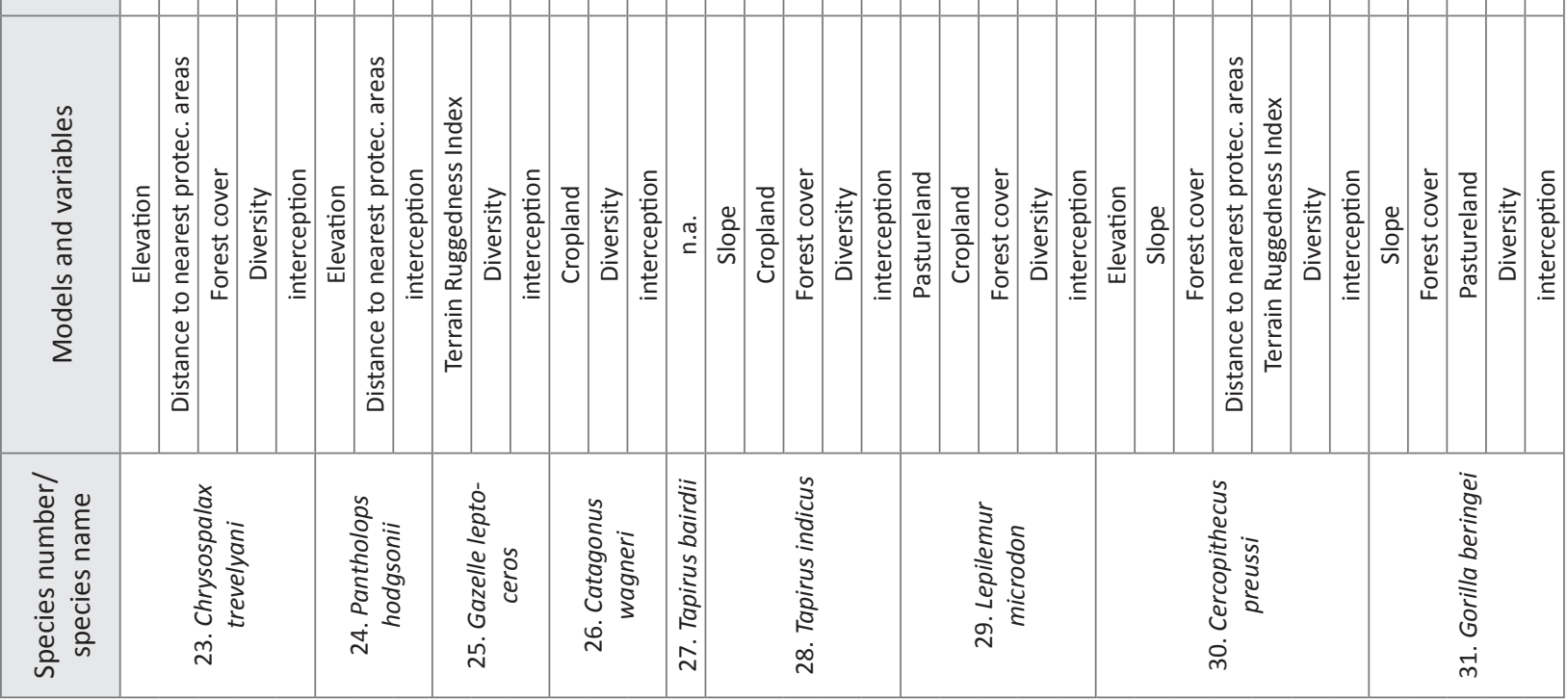




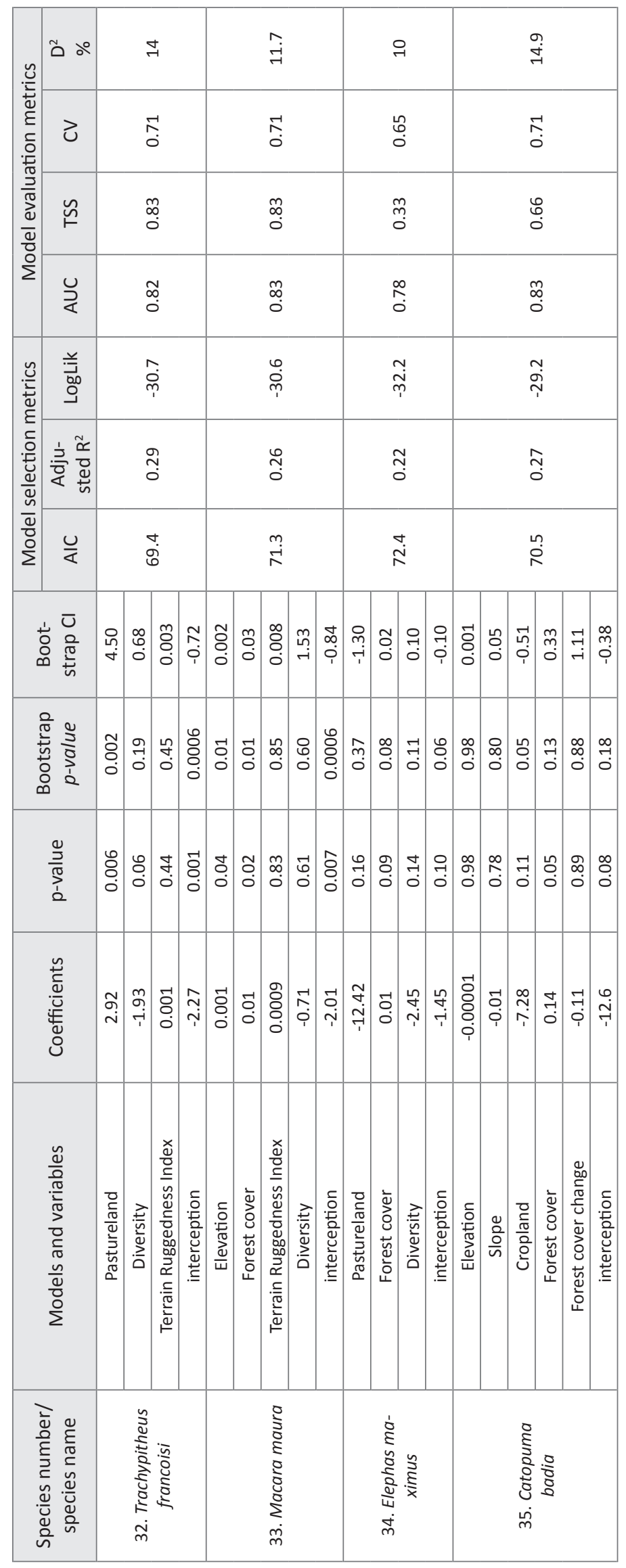



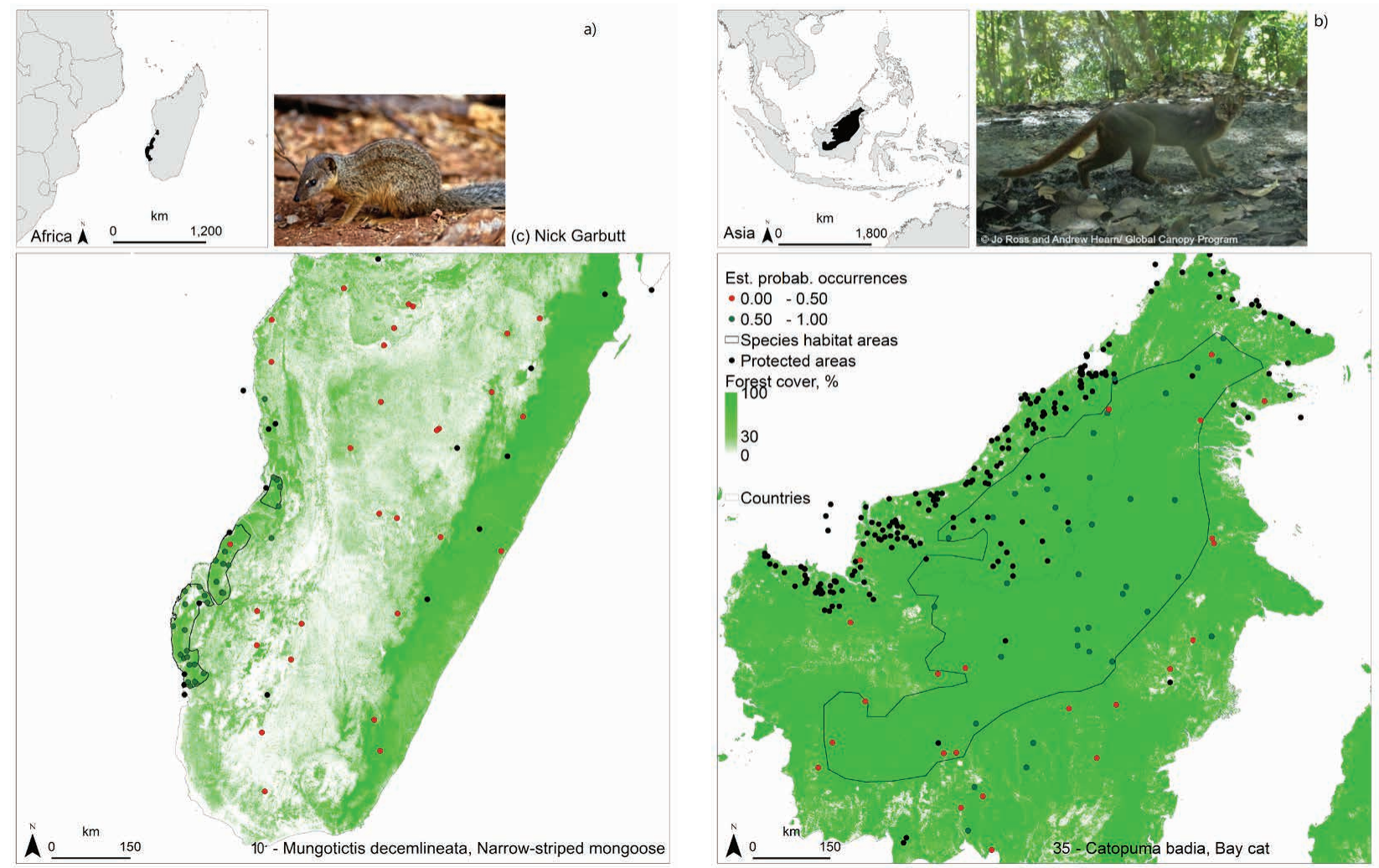

Figure 2: Estimated probability of species occurrence, species habitat areas, forest cover above 30 percent and protected areas of (a) 10- Mungotictis decemlineata, (b) 35-Catopuma badia

topuma badia, forest cover change affected negatively to the estimated probability of occurrence records of this large-body mammal. Estimated probability of species occurrence records (and pseudo-absences above $\mathrm{H}>0.50$; Figure 2) tended to respectively occupy dense forest cover above 89 percent and 27 percent for Catopuma badia and Mungotictis decemlineata.

Estimated probability of species occurrences tended to be near the protected areas in 22 percent of $35 \mathrm{GLM}$ models. In total, 45 percent of mammal species including endangered mammal species of Lontra provocax and Lontra feline occupying vegetated coastal areas were negatively influenced by the expansion of pastureland and or of pastureland and cropland altogether (Table 2).

\subsection{Evaluation of regression model results}

The (average) species occurrence predictions of MaxEnt were higher than the species occurrence predictions ( $H$ in Figure 3 ) obtained from GLM models, for all endangered mammal species. The average species occurrence prediction values obtained (from MaxEnt) using GBIF occurrence data records and randomly selected endangered mammal species occurrences were very similar (above 0.90) (Figure 3).

On an average, 58.7 percent and 57.8 percent of GBIF species occurrence records overlapped with endangered mammal species habitat areas (Figure 4).
Very similar values were found between 10 -fold and 100-fold cross-validation for all species models. In total, 51, 74 and 71 percent of GLM models with one, two and four randomly replaced occurrences (with no occurrences) respectively obtained a higher AIC value (in absolute terms) $(|\Delta \mathrm{AIC}|>2)$ compared to thirty-five endangered mammal species models. In total, 74 and 85 percent of GLM models with randomly replaced one, two and four species occurrences received lower values of ten fold-cross-validation and of AUC compared to thirty-five endangered mammal species model (see 1.4 Model evaluation).

The results of the change of AIC ( $\triangle \mathrm{AIC}$ ), of ten-foldcross-validation and of AUC are shown in Figure S6.

\section{DISCUSSION}

The thirty-five-endangered mammal species were considered in this study because they present a good study case for the other species groups (Di Marco et al., 2017). This study results showed that distributions of thirty-five endangered mammal species were determined by the abundance of environmental resources, high elevation, and the presence of forested landscape. Forest cover, elevation and nearest distance to protected areas were the most significant $(p$-value $<0.05)$ explanatory variables of estimated probability of species occurrence indicating that forested land, topography (elevation) and protected 


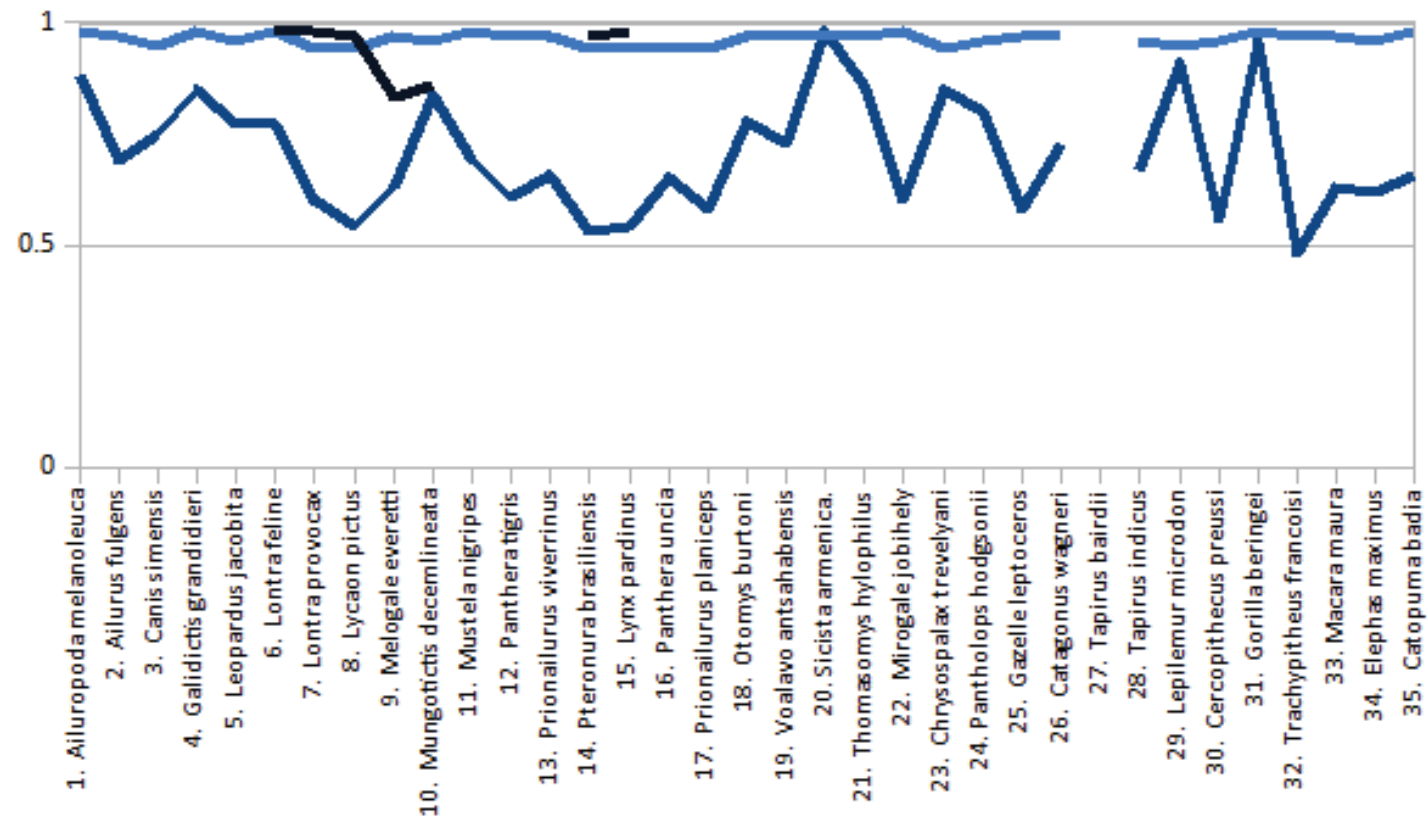

H Presence probability_1 Presence probability_2

Figure 3: The average estimated probability obtained from GLM models and the presence probability of species occurrences obtained from MaxEnt. The average estimated probability $(H)$ is a threshold (average) value of occurrence predictions obtained from GLM models. Presence probability_1 was obtained by using random species occurrences within species habitats obtained from MaxEnt, for thirty-five species. Presence probability_2 was obtained by using GBIF species occurrence records between 2000 and 2015 for six species (> 20 occurrence records) from MaxEnt. There were excluded sixteen species with no GBIF occurrence data records and eleven species with GBIF species occurrence records between one occurrence record and twelve occurrence records. The numbers of GBIF occurrence records are in Table S3. There were no results for species Tapirus bairdii (number twenty-seven)

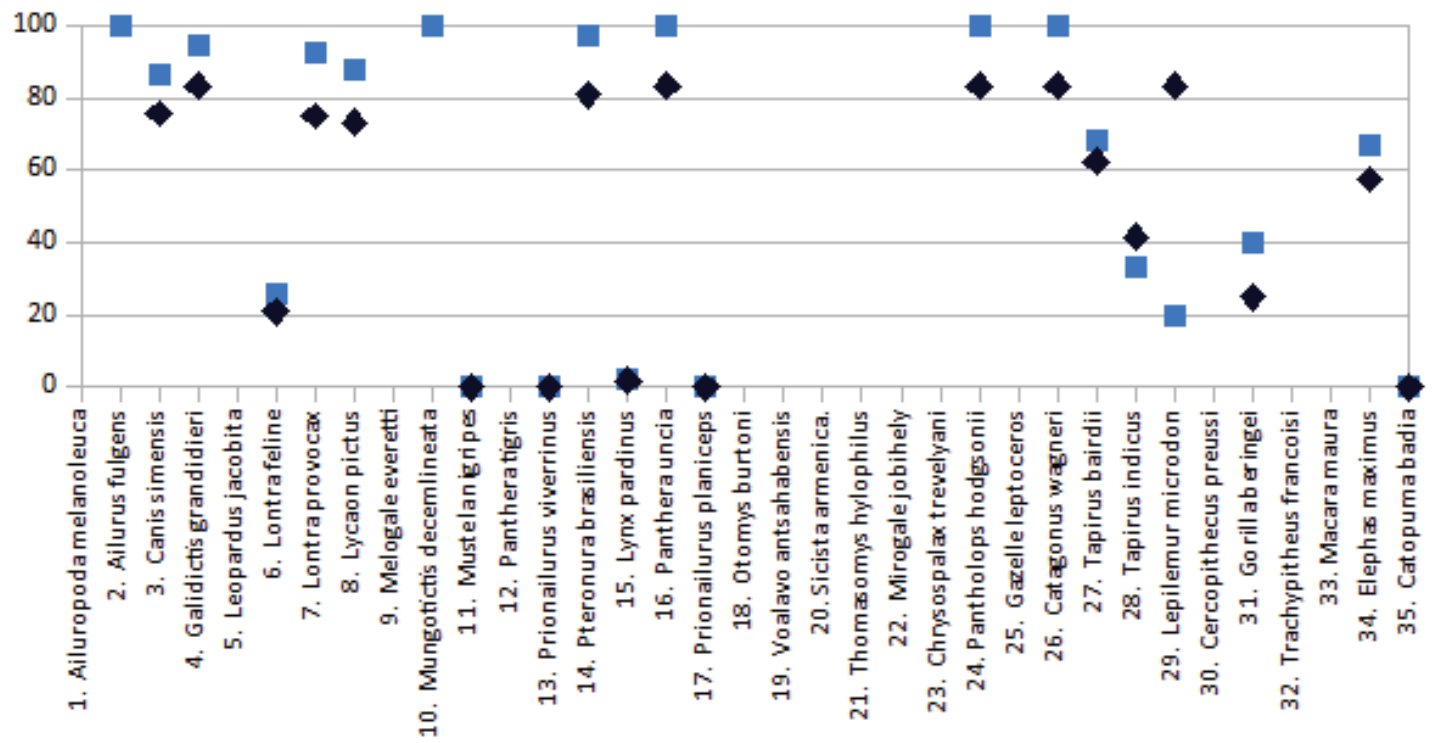

- The percentage of occurrence records overlapped with species habitat areas, between 18th century and 2017 - The percentage of occurrence records overlapped with species habitat areas, between 2000 and 2015

Figure 4: The percentage of GBIF occurrence records between 2000 and 2015 and between 1885 and 2017 overlapped with IUCN endangered mammal species habitat areas. Endangered mammal species number two and twelve had no occurrence records between 2000 and 2015 
forests can strongly determine the endangered mammal species occurrences in their forested habitat areas. Forests were also determined by other studies as key-factors for terrestrial species survival, for example, for Lynx pardinus, Panthera tigris, Ailuropoda melanoleuca and Gorilla beringei (Fernandez et al., 2006; Fernández et al., 2003; Kanagaraj et al., 2011; Yu et al., 2003; Strindberg et al., 2018) and for terrestrial endangered mammal species (IUCN 2016). Terrestrial and semi-aquatic mammal species preferred elevated, rugged and forested landscape in twenty-eight percent of GLM models. The GLM results supported the view that elevated forested land can provide refuge areas for terrestrial and semi-aquatic species. Antunes et al. (2016) found, respectively, that 80 percent and 50 percent of terrestrial and aquatic species (including Pteronura brasiliensis) in Amazon forests refuged into upland forested areas and thus were saved from hunting activities. High topography helps habitat diversity and higher species richness, for example, of plants (Distler et al., 2009), which could be searched for food by endangered mammal species.

The GLM models demonstrated that distributions of large-body carnivore of Catopuma badia and small mammal species of Mungotictis decemlineata can be conditionally distributed by the changes in forests. They showed that estimated high probability of occurrences of Catopuma badia (above 0.66) and Mungotictis decemlineata (above 0.84) in Figure 2 were located in dense forest cover of 96 percent and 34 percent, respectively, within their species habitat areas. The species of Catopuma badia and Mungotictis decemlineata showed clear patterns of areas with high probability of occurrences with respect to a dense forested landscape, indicating that dense forested landscape is a natural resource condition for these species to persist. I note here that the forest cover of thirty percent is a threshold for the diversity of small mammals (Estavillo et al., 2013).

I used GLMs for identifying the areas of high probability of species occurrences in relation to environmental and natural resources and conditions that determine the distribution of the mammal species. The GLM models are used in habitat modelling of large-carnivore species because they are an effective modelling approach for species distribution modelling, which perform well with adequate species data and handling non-linear response between species occurrences and environmental variables (Kanagaraj et al. 2013). Yet, I checked endangered mammal species model results and species assumption on selecting randomly occurrences within endangered mammal species habitat areas for the GLM species models (see 1.1. Endangered species data) by randomly removing a different number of species occurrences and by replacing species occurrences with no species occurrences (see 1.4 Model evaluation). Endangered mammal species models (with replaced occurrences) performed worse than the species models $(\triangle A I C>2)$ in Table 2. About 60 percent of GBIF endangered mammal species occurrences coincides with endangered mammal species habitat areas (of IUCN). Overall, the assumption on the randomly selection of endangered mammal occurrences within endangered mammal species habitat areas was adequate.

\subsection{Data differences}

I used species and forest data collected around the same time in order to reduce the data differences for species and forest cover changes. I used the most relevant species data of the year 2015, which were provided by IUCN and GBIF. Yet, 17 percent and 46 percent of endangered mammal species had respectively less than twelve occurrence records or no GBIF occurrences records being collected between the year of 2000 and 2015. The increased detailed data availability on forest cover changes caused by forest activities (logging, clear-cutting) would improve the predictions of species distributions and the understanding of relationships between the spatial and temporal changes in forest cover and the changes in endangered mammal species occurrences (records). Overall, I argue that there is a need for a standardized monitoring of endangered mammals species data, for gathering of intensive data and evidences on the changes in forested landscape, and of detailed data on negative impacts of any forest activity on forests like clear-cutting, selective logging (selective tree cutting), mechanization use (for tree removal), of forest activity noise, and on the stage of agriculture development. Ester et al. (2018) found that different stages of agriculture landscape (high intensified, low intensified agriculture) determined the distribution of terrestrial mammal species. Strindberg et al. (2018) measured the canopy height (Gorilla beringei species preferred high trees between $25 \mathrm{~m}$ to $35 \mathrm{~m}$ ), to distinguish between selective logging (high trees) and swap forests (shorter trees) and found that tropical forests were degraded, when selective logging was applied. The removal of high trees (> $25 \mathrm{~m}$ ) can possibly change the occurrences of Gorilla beringei species from logged to unlogged (intact) forests. The forest clear-cutting (cutting down all trees in a given area) could dramatically change the Gorilla beringei occurrences pushing this species away from cleared forested area. This should be investigated for Catopuma Badia that was negatively impacted from the forest cover changes. In addition, any removal tree activity is associated with noise due to mechanization used in forests. High noise levels may disturb endangered mammal species. Buxton et al. (2017) estimated high noise levels in protected areas (in USA) and found that anthropological noise caused by extraction activities of land use (e.g., logging) disturbed endangered species in the protected areas.

The endangered mammal species model results clearly demonstrated that the expansion of cropland and or pastureland to forested habitat was associated with fewer estimated probability of endangered mammal species occurrence (Table 2). This was in line with the findings of Mohd-Azlan and Sanderson (2007), who found that the changes in land use, for example, from forests to cropland, were one of the threats to Catopuma badia carnivore species. In line with the studies of endangered species that were threatened by high-intensity land use (Pekin \& Pijanowski 2012), the findings of this study 
support the view that fewer estimated probability of species occurrence in cropland and or pastureland were the result of the expansion of cropland and or pastureland land on forests and shrub lands. Forested landscape modification caused by the expansion of cropland (and or pastureland) to forested land was one of the key drivers of global species loss (Fischer \& Lindenmayer, 2007). Ester et al. (2018) found that intensified agricultural landscapes threaten worldwide terrestrial mammal species, while the expansion of low intensity agriculture can be considered as a threat (to the Wildness Areas including mammals), if the agricultural expansion occurs in the future (Veach et al., 2017).

\subsection{Role of protected areas and of cross-border areas}

Protected areas are a key-solution for conserving biodiversity throughout the world (DeFries et al., 2007; Pollock et al., 2017), for protecting species habitat due to the implementation of wildlife laws. Strindberg et al. (2018) showed that a higher density population of endangered species of gorillas including Gorilla beringei and chimpanzees were found in protected areas and where wildlife laws were enforced. Panda, a symbol of an endangered species, was formerly distributed over a larger area including China and its cross-border areas with Vietnam and Burma, but today, this species can be found only in few provinces in China as result of hunting, logging and clearing for agriculture (MacKinnon \& De Wulf, 1994). Protected areas were then designed to save this species (Liu et al., 2001). After a few decades, the classification of the endangered species of panda changed from a 'threaten' species to a 'vulnerable' species in the year 2016 (www.iucnredlist.org).

To achieve Aichi Target 11 for protecting biodiversity in the future (Pollock et al., 2017), countries can establish and upgrade (new) protected areas. The expansion of new protected areas could improve mammal diversity (Pollock et al., 2017) for all countries involved in species conservation. Protected areas can be expanded and or can be newly established in the surrounding of the existing protected areas (in crossborder areas or in country) to reduce the human pressure on protected areas and their edges. Almost all carnivore species were not present at the edges of protected areas and carnivore diversity was higher in protected areas with low human disturbance (Baker \& Leberg, 2018). This study showed that estimated probability of occurrences for twenty-two percent of endangered mammal species, for example, Mungotictis decemlineata (Table 2), tended to be located in the proximity of the protected areas, indicating that the surroundings of protected areas could also be effective in species conservation and protection (e.g., DeFries et al., 2007; Knorn et al., 2012). The surrounding areas of the protected areas can be (surrounding) human land use that can maintain source habitat of species

Percentage of forest cover for endangered mammal species

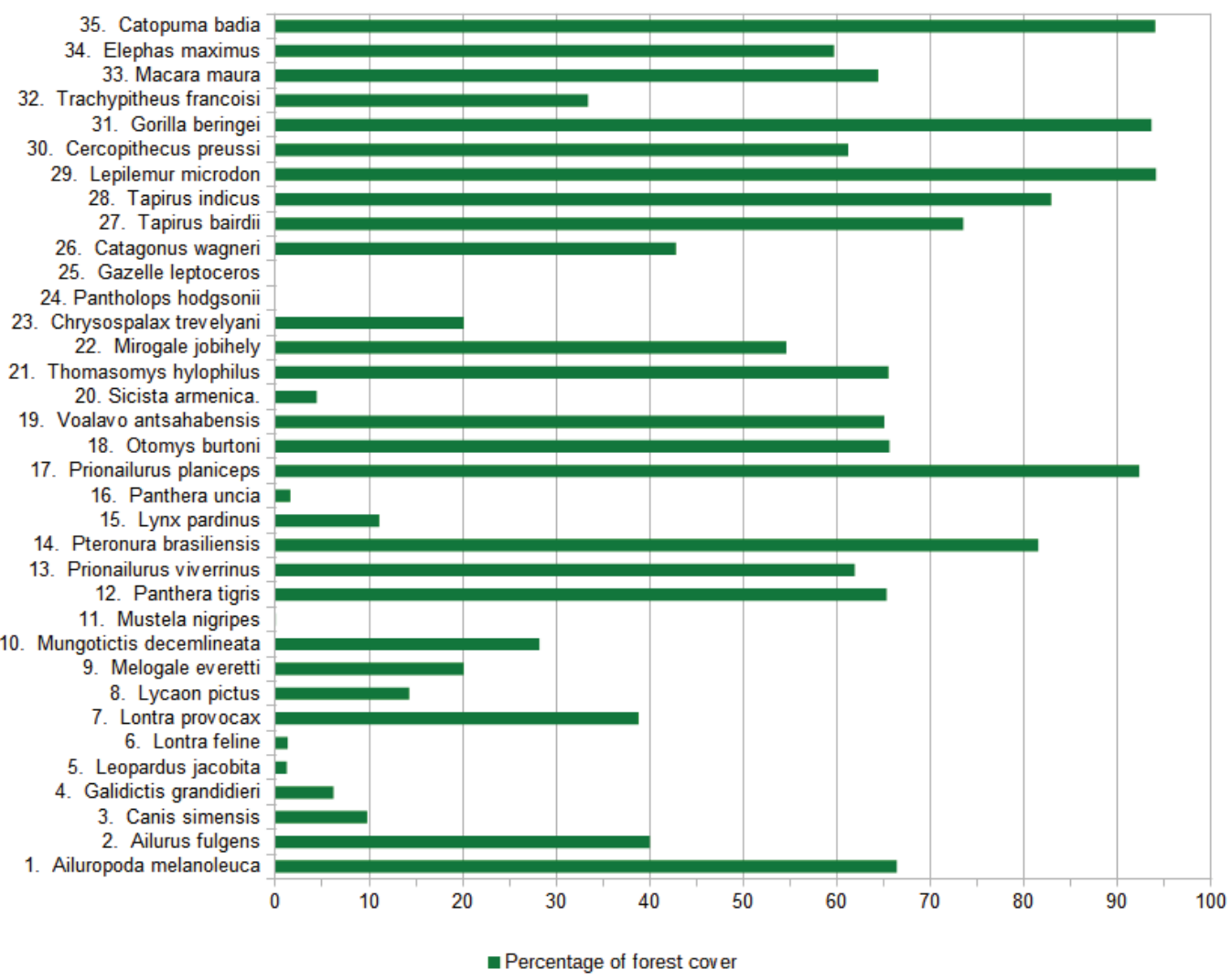

Figure 5: The percentage of forest cover in the year 2000 for endangered mammal species. Forest cover data were provided by Hansen et al 2013.] 
(Hansen \& DeFries, 2007), though there is a strong need for better understanding of the sensitivity of species towards local human land use, particularly, towards old-growth forests and secondary and species-rich secondary forests that vary in tree species (forest) composition (see Rozendaal et al., (2019)). New protected areas can also be established outside the protected areas, where there is a considerable population of endangered mammal species. For example, Strindberg et al (2018) found that around 80 percent of the endangered species of gorillas including Gorilla beringei and chimpanzees in Africa were found outside of the protected areas occupying landscape with intact forests. This would require enforcement to protect the gorillas and chimpanzees population and intact forests. Here, new protected areas could be established where forest cover is above thirty percent for small mammal species diversity (Estavillo et al., 2013), above fifty percent for primates as shown by the forest data (species numbered from 29 to 31 and 33 in Figure 5), as well as by studies on mammal species of Nüchel et al. (2018), and on gorillas including Gorilla beringei and chimpanzees of Strindberg et al. (2018).

An efficient management of protected areas would require new data collection on forests, on endangered mammal species and on their species preys. The gathering of new data and of new scientific evidences could not miss areas with higher prey densities that is attractive to carnivore endangered species for searching food, for example, Panthera tigris altaica (Carroll \& Miquelle 2006). This can help in identifying new areas with large( $r$ ) population of endangered mammal species inhabiting intact or less disturbed forests.

The thirty-seven percent of endangered mammal species were protected by IUCN protected areas categories from one to four (Table S3). The forty-five percent of endangered mammal species had a shared species habitat area amongst countries. The categories of protected areas (applied to endangered mammal species habitats) varied amongst countries. This means that the management and enforcement changed from one country to another by potentially affecting species survival. To efficiently conserve endangered mammal species and forests in their cross-border areas countries may apply a regional approach (lojă et al., 2010) by (re)establishing protected areas preferably of the same high protection categories (from one to four) of IUCN. This cannot only help countries unify the management and enforcement in their protected cross-border areas, but also help them increase gains in forest cover and in carbon sequestration (Viña and Liu, 2017) affecting the climate.

\section{CONCLUSIONS}

Against the extinction of threatened species, alternatives are drawn to conserve species. Many studies focus on calculating the conservation areas for species and proposing the creation of new protected areas to conserve threaten animal species. Protection of forested landscape and upland forests either in cross-border areas or in countries can be a conservation option for terrestrial and semi-aquatic endangered mammal species. I conclude that conservation of endangered mammal species may potentially need an effective regional approach to be applied by the countries supported by continuous and standardized monitoring of species and forest data collection as well as by updated scientific evidences to better conserve terrestrial and semi-aquatic endangered mammal species and mammal diversity in forests.

\section{References}

Allouche, O., Tsoar, A. \& Kadmon, R., 2006. Assessing the accuracy of species distribution models: prevalence, kappa and the true skill statistic (TSS). Journal of Applied Ecology, 43(6), pp.1223-1232. Available at: https://besjournals.onlinelibrary.wiley.com/doi/ abs/10.1111/j.1365-2664.2006.01214.x.

Anselin, L., 1988. Spatial Econometrics: Methods and Models, Dordrecht: Kluwer Academic Publishers.

Anselin, L., Syabri, I. \& Kho, Y., 2006. GeoDa: An Introduction to Spatial Data Analysis. Geographical Analysis 38 (1), 5-22.

Baker, A.D. \& Leberg, P.L., 2018. Impacts of human recreation on carnivores in protected areas. PLOS ONE, 13(4), pp. 1-21. Available at: https://doi.org/10.1371/journal.pone.0195436.

Barnosky, A.D. et al., 2016. Variable impact of late-Quaternary megafaunal extinction in causing ecological state shifts in North and South America. Proceedings of the National Academy of Sciences of the United States of America, 113(4), pp. 856-861. Available at: http://www.ncbi.nlm.nih.gov/pmc/articles/PMC4739530/.

Baselga, A. et al., 2012. Global patterns in the shape of species geographical ranges reveal range determinants. Journal of Bio- geography, 39(4), pp. 760-771. Available at: http://dx.doi. org/10.1111/j.1365-2699.2011.02612.x.

Buxton, R.T. et al., 2017. Noise pollution is pervasive in U.S. protected areas. Science, 356(6337), pp. 531-533. Available at: http://science.sciencemag.org/content/356/6337/531.

Carroll, C. \& Miquelle, D.G., 2006. Spatial viability analysis of Amur tiger Panthera tigris altaica in the Russian Far East: the role of protected areas and landscape matrix in population persistence. Journal of Applied Ecology, 43(6), pp. 1056-1068. Available at: http://dx.doi.org/10.1111/j.1365-2664.2006.01237.x.

Ceballos, G. \& Ehrlich, P.R., 2006. Global mammal distributions, biodiversity hotspots, and conservation. Proceedings of the National Academy of Sciences, 103(51), pp. 19374-19379. Available at: http://www.pnas.org/content/103/51/19374.abstract.

Chefaoui, R.M. \& Lobo, J.M., 2008. Assessing the effects of pseudo-absences on predictive distribution model performance. Ecological modelling, 210(4), pp. 478-486. 
Conrad, O. et al., 2015. System for Automated Geoscientific Analyses (SAGA) v. 2.1.4. Geoscientific Model Development, 8(7), pp. 1991-2007.

DeFries, R. et al., 2007. Land use change around protected areas: Management to balance human needs and ecological function. Ecological Applications, 17(4), pp. 1031-1038. Available at: http:// dx.doi.org/10.1890/05-1111.

Distler, T. et al., 2009. Determinants and prediction of broad-scale plant richness across the western neotropics. Annals of the Missouri Botanical Garden, 96(3), pp. 470-491. Available at: http:// dx.doi.org/10.3417/2008034.

Dormann, C.F., 2007. Effects of incorporating spatial autocorrelation into the analysis of species distribution data. Global Ecology and Biogeography, 16(2), pp. 129-138. Available at: http://dx.doi. org/10.1111/j.1466-8238.2006.00279.x.

Elith, J. \& Leathwick, J.R., 2009. Species Distribution Models: Ecological Explanation and Prediction Across Space and Time. Annual Review of Ecology, Evolution, and Systematics, 40(1), pp. 677-697. Available at: https://doi.org/10.1146/annurev.ecolsys.110308.120159.

Environmental Systems Research Institute, 2016. ArcGIS Release 10.3. Redlands, CA.

Estavillo, C., Pardini, R. \& Rocha, P.L.B. da, 2013. Forest Loss and the Biodiversity Threshold: An Evaluation Considering Species Habitat Requirements and the Use of Matrix Habitats. PLOS ONE, 8(12), p.e82369. Available at: http://dx.doi.org/10.1371\%2Fjournal. pone.0082369.

Ester, P. et al., 2018. From tropical shelters to temperate defaunation: The relationship between agricultural transition stage and the distribution of threatened mammals. Global Ecology and Biogeography, 27(6), pp. 647-657. Available at: https://onlinelibrary. wiley.com/doi/abs/10.1111/geb.12725.

Faurby, S. \& Svenning, J.C., 2015. Historic and prehistoric human-driven extinctions have reshaped global mammal diversity patterns. Diversity and Distributions, 21(10), pp. 1155-1166. Available at: http://dx.doi.org/10.1111/ddi.12369.

Feng, G. et al., 2017. Historical anthropogenic footprints in the distribution of threatened plants in China. Biological Conservation, 210, pp. 3-8.

Fernández, N. et al., 2003. Identifying breeding habitat for the Iberian lynx: Inferences from fine-scale spatial analysis. Ecological Applications, 13(5), pp. 1310-1324. Available at: http://dx.doi. org/10.1890/02-5081.

Fernandez, N., Delibes, M. \& Palomares, F., 2006. Landscape evaluation in conservation: molecular sampling and habitat modeling for the Iberian lynx. Ecological applications : a publication of the Ecological Society of America, 16(3), pp. 1037-1049.

Fischer, G. et al., 2008. Global Agroecological Zones Assessment for Agriculture (GAEZ 2008), Laxenburg. Austria. Available at: www. fao.org.

Fischer, J. \& Lindenmayer, D.B., 2007. Landscape modification and habitat fragmentation: a synthesis. Global Ecology and Biogeography, 16(3), pp. 265-280. Available at: http://dx.doi. org/10.1111/j.1466-8238.2007.00287.x.
Foley, J.A. et al., 2005. Global Consequences of Land Use. Science, 309(5734), pp. 570-574. Available at: http://www.sciencemag. org/cgi/content/abstract/309/5734/570.

Gaillard, J.M. et al., 2010. Habitat-performance relationships: finding the right metric at a given spatial scale. Philos Trans $R$ Soc Lond B Biol Sci, 365(1550), pp. 2255-2265.

GBIF.org, 2018a. GBIF Occurrence Download. Downloaded on 13 January 2018. https://doi.org/10.15468/dl.gfufyq.

GBIF.org, 2018b. GBIF Occurrence Download. Downloaded on 13 January 2018. https://doi.org/10.15468/dl.jgy1ug.

Hansen, A.J. \& DeFries, R., 2007. Ecological mechanisms linking protected areas to surrounding lands. Ecological Applications, 17(4), pp. 974-988. Available at: http://dx.doi.org/10.1890/05-1098.

Hansen, M.C. et al., 2013. High-resolution global maps of 21st-century forest cover change. Science (New York, N.Y.), 342(6160), pp. 850-853.

Hesterberg, T.C., 2015. What Teachers Should Know About the Bootstrap: Resampling in the Undergraduate Statistics Curriculum. The American Statistician, 69(4), pp. 371-386. Available at: https://doi.org/10.1080/00031305.2015.1089789.

Hosmer, D.W. \& Lemeshow, S., 2000. Applied Logistic Regression, John Wiley and Sons (ed.), New York, USA, 373pp. Available at: http:// ecsocman.edu.ru/db/msg/2425.html.

Iojă C. I. et al., 2010. The efficacy of Romania's protected areas network in conserving biodiversity. Biological conservation, 143:2468-2.

IUCN, 2012. IUCN Red List Categories and Criteria: Version 3.1. Second edition., Gland, Switzerland and Cambridge, UK: IUCN. iv + 32pp. Available at: http://s3.amazonaws.com/iucnredlist-newcms/ staging/public/attachments/3097/redlist_cats_crit_en.pdf.

IUCN, 2017. IUCN Spatial Data Resources. Available at: http://www. iucnredlist.org/technical-documents/red-list-training/iucnspatialresources [Accessed October 18, 2016].

IUCN, 2016. The IUCN Red List of Threatened Species. Version 2016-2. Available at: http://www.iucnredlist.org/technical-documents/ spatial-data [Accessed October 18, 2016].

IUCN and UNEP-WCMC, 2015. The World Database on Protected Areas (WDPA) [Online], [version July 2015], Cambridge, UK:UNEPWCMC. Available at: www.protectedplanet.net. [Accessed May $3,2016]$.

Johnson, C.J., Seip, D.R. \& Boyce, M.S., 2004. A quantitative approach to conservation planning: using resource selection functions to map the distribution of mountain caribou at multiple spatial scales. Journal of Applied Ecology, 41(2), pp. 238-251. Available at: http://dx.doi.org/10.1111/j.0021-8901.2004.00899.x.

Kanagaraj, R. et al., 2011. Assessing habitat suitability for tiger in the fragmented Terai Arc Landscape of India and Nepal. Ecography, 34(6), pp. 970-981. Available at: http://dx.doi.org/10.1111/ j.1600-0587.2010.06482.x.

Kehoe, L. et al., 2016. Agriculture rivals biomes in predicting global species richness. Ecography, p.n/a-n/a. Available at: http://dx.doi. org/10.1111/ecog.02508.

Knorn, J. et al., 2012. Forest restitution and protected area effectiveness in post-socialist Romania. Biological Conservation, 146(1), pp. 204-212. Available at: http://www.sciencedirect.com/science/article/pii/S0006320711004836. 
Liu, C. et al., 2005. Selecting thresholds of occurrence in the prediction of species distributions. Ecography, 28(3), pp. 385-393. Available at: http://dx.doi.org/10.1111/j.0906-7590.2005.03957.x.

Liu, J. et al., 2001. Ecological Degradation in Protected Areas: The Case of Wolong Nature Reserve for Giant Pandas. Science, 292(5514), pp. 98-101.

Mackinnon, J. \& De Wulf, R., 1994. Designing protected areas for giant pandas in China. In R. I. Miller, ed. Mapping the Diversity of Nature. Dordrecht: Springer Netherlands, pp. 127-142. Available at: http://dx.doi.org/10.1007/978-94-011-0719-8_8.

Di Marco, M. et al., 2017. Limitations and trade-offs in the use of species distribution maps for protected area planning. Journal of Applied Ecology, 54(2), pp. 402-411. Available at: http://dx.doi. org/10.1111/1365-2664.12771.

McCullagh, P. \& Nelder, J.A., 1989. Generalized Linear Models. Chapman and Hall, London, England, $511 \mathrm{pp}$.

Mohd-Azlan, J. \& Sanderson, J., 2007. Geographic distribution and conservation status of the bay cat Catopuma badia, a Bornean endemic. Oryx, 41(3), pp. 394-397. Available at: https://www. cambridge.org/core/article/geographic-distribution-and-conservation-status-of-the-bay-cat-catopuma-badia-a-bornean-endemic/F80BFBE97BE28558BCAA3173F5A343E3.

Naves, J. et al., 2003. Endangered Species Constrained by Natural and Human Factors: The Case of Brown Bears in Northern Spain. Conservation Biology, 17(5), pp. 1276-1289. Available at: http:// www.jstor.org/stable/3588953.

Nüchel, J. et al., 2018. Snub-nosed monkeys (Rhinopithecus): potential distribution and its implication for conservation. Biodiversity and Conservation, 27(6), pp. 1517-1538. Available at: https:// doi.org/10.1007/s10531-018-1507-0.

Pekin, B.K. \& Pijanowski, B.C., 2012. Global land use intensity and the endangerment status of mammal species. Diversity and Distributions, 18(9), pp. 909-918. Available at: http://dx.doi. org/10.1111/j.1472-4642.2012.00928.x.

Phillips, S.J., Anderson, R.P. \& Schapire, R.E., 2006. Maximum entropy modeling of species geographic distributions. Ecological Modelling, 190(3-4), pp. 231-259.

Pimm, S.L. \& Raven, P., 2000. Biodiversity: Extinction by numbers. Nature, 403(6772), pp.843-845. Available at: http://dx.doi. org/10.1038/35002708.
Pollock, L.J., Thuiller, W. \& Jetz, W., 2017. Large conservation gains possible for global biodiversity facets. Nature, 546(7656), pp. 141-144.

R Development Core Team, 2018. Bootstrap Resampling for Mixed Effects and Plain Models. Available at: github.com/ColmanHumphrey/glmmboot.

R Development Core Team, R., 2011. R: A Language and Environment for Statistical Computing R. D. C. Team, ed. R Foundation for Statistical Computing, 1(2.11.1), p. 409. Available at: http://www.rproject.org.

Ramankutty, N. et al., 2010. Global Agricultural Lands: Pastures, 2000. Data distributed by the Socioeconomic Data and Applications Center (SEDAC). Available at: http://sedac.ciesin.columbia.edu/ es/aglands.html [Accessed May 26, 2016].

Rogers, L.M. \& Gorman, M.L., 1995. The population dynamics of small mammals living in set-aside and surrounding semi-natural and crop land. Journal of Zoology, 236(3), pp. 451-464. Available at: http://dx.doi.org/10.1111/j.1469-7998.1995.tb02724.x.

Rozendaal, D. M. A. et al., 'Biodiversity recovery of Neotropical secondary forests,' Sci. Adv., vol. 5, no. 3, p. eaau3114, Mar. 2019.

Soto, C. \& Palomares, F., 2015. Coexistence of sympatric carnivores in relatively homogeneous Mediterranean landscapes: functional importance of habitat segregation at the fine-scale level. Oecologia, 179(1), pp. 223-235.

Strindberg, S. et al., 2018. Guns, germs, and trees determine density and distribution of gorillas and chimpanzees in Western Equatorial Africa. Science Advances, 4(4). Available at: http://advances. sciencemag.org/content/4/4/eaar2964.abstract.

Veach, V., Moilanen, A. \& Di Minin, E., 2017. Threats from urban expansion, agricultural transformation and forest loss on global conservation priority areas. PLOS ONE, 12(11), p.e0188397. Available at: https://doi.org/10.1371/journal.pone.0188397.

Viña, A. and Liu, J. (2017) 'Hidden roles of protected areas in the conservation of biodiversity and ecosystem services', Ecosphere, 8(6), p. ecs2.1864. doi: 10.1002/ecs2.1864.

Wich, S.A. et al., 2014. Will Oil Palm's Homecoming Spell Doom for Africa's Great Apes? Current Biology, 24(14), pp. 1659-1663. Available at: http://dx.doi.org/10.1016/j.cub.2014.05.077.

Yu, G. et al., 2003. Feeding habitat of giant pandas (Ailuropoda melanoleuca): why do they prefer bamboo patch edges? Journal of Zoology, 261(3), pp. 307-312. Available at: https://www. cambridge.org/core/article/feeding-habitat-of-giant-pandasailuropoda-melanoleuca-why-do-they-prefer-bamboo-patchedges/78CB23D3484FBB2EC43222CAEB126EDE. 


\section{SUPPLEMENTARY MATERIAL}

\section{S1. Species habitat areas}

For every endangered mammal species, the information was collected on species classification, geographical range, red list category and criteria, habitat preferences, major threats, conservation measures, species utilisation (https://iucnredlist.org/ initiatives/mammals/process/methods). A distribution map was available (polygon) that consisted of more than one polygon where there was a discontinuity in a species suitable habitat, for every endangered mammal species (IUCN 2016).

Species had different habitat areas with the smallest habitat area of Melogale everetti $\left(20.6 \mathrm{~km}^{2}\right)$ and the largest habitat area of Pteronura brasiliensis $\left(7100 \mathrm{~km}^{2}\right)$, Table S3. The largest area of 94 percent covered by forests was found for Lepilemur microdon and Catopuma badia followed by Gorilla beringei with a forest cover area of 93 percent. The greatest forest cover loss of 19.7 percent was calculated for Tapirus indicus species. The largest area covered by pastureland and cropland was respectively found for Mustela nigripes, 80.5 percent and for Macara maura, 43.1 percent. The habitat areas of the species of Ailuropoda melanoleuca, Canis simensis and Lontra feline were divided into more than 10 smaller habitat areas. The species habitat area protection varied amongst countries in terms of the number of established protected areas (point data) within a cross-border species habitat area. For example, the species habitat areas of Pteronura brasiliensis were not protected (in the cross-border areas) by all countries in South America. There was found a discrepancy in the numbers of established protected areas for the species of Elephas maximus in 14 countries located in Asia. In total, 37 percent and 63 percent of (35) endangered species were protected by IUCN category (maps.iucnredlist.org) between one and four (higher protection) and between five, six and unknown (lower protection). Here, we note that all protected areas are not explicitly established for the endangered mammal species conservation (Table S3).

\section{S2. Characteristics of species habitat area}

1. Ailuropoda melanoleuca. The species habitat area was divided into twenty smaller areas (twenty polygons) situated in an averaged elevated land of over $2000 \mathrm{~m}$ above sea level and in a forested land area that was protected. 2. Ailurus fulgens. This species had a cross-border habitat area. The species habitat area was not divided into smaller areas (it was one single polygon). The species habitat area was located at very high elevated areas, in forested areas and was protected. 3. Canis simensis. The species habitat area was divided into twelve smaller areas (twelve polygons) placed in low elevation with few forests in it and it was not protected. 4. Galidictis grandidieri. The species habitat area was located close to a coastal area with few forests in it, though it was not divided and not protected. 5. Leopardus jacobita. The species habitat area was divided into one large area and three small ones situated in high elevated land with limited forests protection. 6 . Lontra feline. The species habitat area was divided into 11 smaller habitats along the coast. This was a cross-border species habitat area and was not protected. 7. Lontra provocax. The species habitat area was divided into one large area and seven smaller ones. This coastal species habitat area was shared amongst countries. 8. Lycaon pictus. The species habitat area was divided into small areas across twenty-two countries with some cross-border habitat areas. The species habitat had a low forest cover. The species protection varied across countries, yet, most of the countries tend not to protect this carnivore species. 9. Melogale everetti. The species habitat area was a one small patch on coastal area with some forests in it and was not protected. 10. Mungotictis decemlineata. The species habitat area was divided into three habitat areas on coastal forested area with a limited number of protected areas inside of the southern habitat. 11. Mustela nigripes. This species was introduced into three small habitat areas at elevated land with some forests in it. There were no protected areas within the species habitat areas. 12. Panthera tigris. The species habitat area is divided into smaller areas inside and in cross-border areas. The species habitat areas were in twelve countries. Not all habitat areas were protected by twelve countries. 13. Prionailurus viverrinus. The species habitat area was divided amongst eleven countries and there were cross-border species habitat areas. The species habitat areas tended to be forested land, but not well-protected by all countries. 14 . Pteronura brasiliensis. This was one large cross-border habitat area situated in dense forested areas shared by ten countries. This habitat area was not protected by all countries. 15. Lynx pardinus. Habitat area was divided into two smaller areas with some forests in it. The species habitat area was not protected in the eastern habitat area. 16. Panthera uncia. The species habitat areas were cross-border species habitat area shared by fifteen countries. The species habitat areas had sparse vegetation. The species protection varied amongst countries. 17. Prionailurus planiceps. The species habitat area was placed in islands with dense forests with some parts on coastal surfaces. The species habitat was divided amongst four countries showing differences in species habitat protection. 18. Otomys burtoni. The species habitat was one (one polygon) forested area, but not protected. 19. Voalavo antsahabensis. This species habitat area was one habitat area covered by forests and not protected. 20. Sicista armenica. This was one single habitat area placed in high elevation with little forests and not protected. 21. Thomasomys hylophilus. This was a cross-border species habitat area (shared by two countries) covered by forests at high elevation and not protected. 22. Mirogale jobihely. The species habitat area was one habitat forested area, and not protected. 23. Chrysospalax trevelyani. The species habitat was one habitat located on a coastal area with some forests in it. The species habitat was not protected. 24. Pantholops hodgsonii. The species habitat was one large habitat area with sparse vegetation and it was partially protected. 25. Gazelle leptoceros. The species habitat area was divided into one big and four smaller areas in a desert land. The big habitat area was protected. 26. Catagonus wagneri. The species habitat area enriched with forests was one 
cross-border habitat area that was shared by three countries. This species habitat was protected by two countries. 27. Tapirus bairdii. The species habitat area was shared amongst seven countries. The species habitat areas were located in forested land and on coastal areas. 28. Tapirus indicus. The species habitat areas were divided into smaller areas shared amongst four countries. There were found large differences in species protection. 29. Lepilemur microdon. The species habitat area was one habitat area located in very dense forests at high elevation, and not protected. 30. Cercopithecus preussi. The species habitat areas were divided into one larger cross-border species habitat area and seven smaller habitat areas. The species habitat areas were situated in forests with a limited protection. There were no protected areas in the cross-border species habitat area. 31. Gorilla beringei. The species habitat area was divided into one large area and five small areas. The species habitat areas were in dense forests, but they (species habitats) were not protected. 32. Trachypitheus francoisi. The species habitat area was divided into two large habitats and nine small areas. This was a cross-border habitat area. Forests were preferred by species. There were well-protected species habitat areas. 33. Macara maura. The species habitat area was one habitat area located in the forested area with limited protection. 34. Elephas maximus. The species habitat area was a large habitat area shared by fourteen countries. The species habitat areas were enriched with forests in cross-border areas. There was found a large discrepancy in the protection amongst the countries. 35. Catopuma badia. The species habitat area was one large crossborder habitat area rich of forests. The species habitat area was protected, but the number of protected areas differed by 90 percent and 10 percent between two countries. 


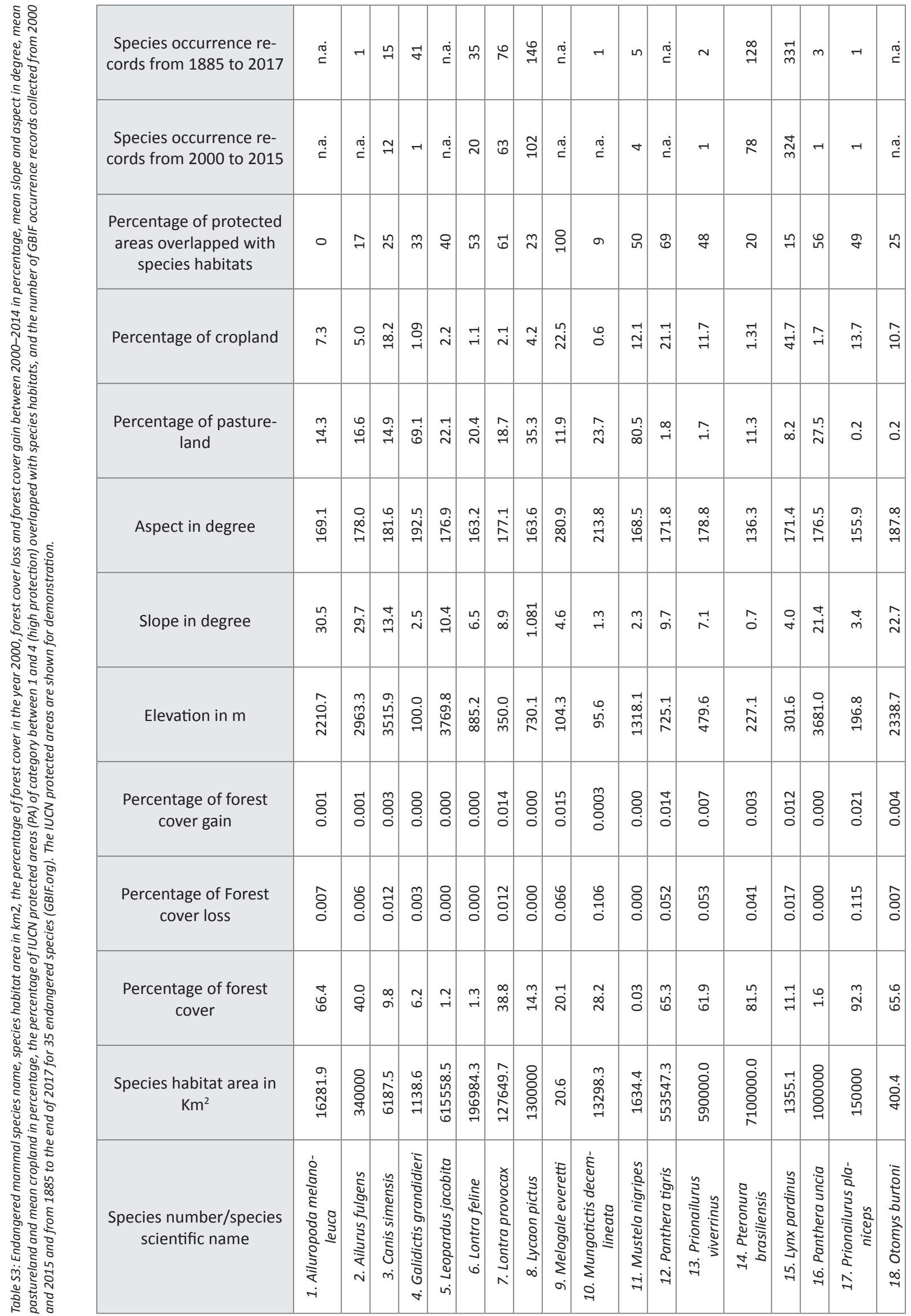




\begin{tabular}{|c|c|c|c|c|c|c|c|c|c|c|c|c|c|c|c|c|c|}
\hline $\begin{array}{l}\text { Species occurrence re- } \\
\text { cords from } 1885 \text { to } 2017\end{array}$ & 官 & 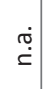 & 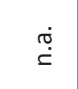 & 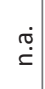 & $\stackrel{\text { ஸேं }}{\check{c}}$ & -1 & $\stackrel{\text { வ் }}{\check{~}}$ & $\nabla$ & $\underset{\substack{\infty \\
\rightarrow}}{\sim}$ & $m$ & in & 官 & $\stackrel{\sim}{\sim}$ & 官 & 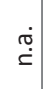 & $\underset{\sim}{\stackrel{\sim}{\sim}}$ & -1 \\
\hline $\begin{array}{l}\text { Species occurrence re- } \\
\text { cords from } 2000 \text { to } 2015\end{array}$ & 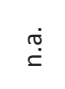 & 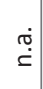 & 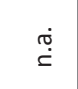 & 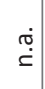 & $\stackrel{\text { ஸ் }}{\check{~}}$ & -1 & 官 & -1 & $\underset{m}{\sim}$ & $\sim$ & $r$ & 官 & 익 & 官 & 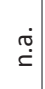 & $\stackrel{\infty}{\infty}$ & -1 \\
\hline $\begin{array}{l}\text { Percentage of protected } \\
\text { areas overlapped with } \\
\text { species habitats }\end{array}$ & 0 & 0 & 운 & 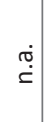 & 0 & 0 & 官 & 윰 & $\vec{m}$ & ఫु & 운 & $\stackrel{\text { ฺேं }}{\check{c}}$ & นก & 官 & 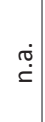 & ถ⿱一𫝀口 & กี \\
\hline Percentage of cropland & $\underset{\sim}{\sim}$ & $\stackrel{m}{\sim}$ & 우 & $\stackrel{m}{n}$ & $\stackrel{\stackrel{n}{-}}{\underset{H}{ت}}$ & $\ddot{0}$ & $\stackrel{l}{0}$ & $\begin{array}{l}\varphi \\
\dot{0}\end{array}$ & $\underset{\rightarrow}{\stackrel{\leftrightarrow}{-}}$ & $\underset{\rightarrow}{\stackrel{-}{*}}$ & $\stackrel{-1}{m}$ & $\begin{array}{l}\sigma \\
\infty \\
\sim\end{array}$ & $\stackrel{+}{-i}$ & $\stackrel{\text { ก̊ }}{\text { gे }}$ & $\vec{m}$ & $\stackrel{-1}{\sim}$ & ન્ન \\
\hline $\begin{array}{l}\text { Percentage of pasture- } \\
\text { land }\end{array}$ & $\stackrel{-1}{\infty}$ & $\stackrel{\infty}{\underset{N}{N}}$ & $\begin{array}{l}\circ \\
\stackrel{\infty}{m}\end{array}$ & $\begin{array}{l}\infty \\
\dot{i} \\
\dot{n}\end{array}$ & $\stackrel{-1}{i}$ & 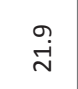 & $\stackrel{\sim}{\rightarrow}$ & $\stackrel{\sim}{i}$ & 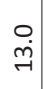 & ָ̃. & \& & $\stackrel{\bullet}{0}$ & $\begin{array}{l}0 \\
\dot{0}\end{array}$ & $\stackrel{m}{f}$ & $\begin{array}{l}\infty \\
\dot{m}\end{array}$ & $\stackrel{\sim}{\sim}$ & ¿ \\
\hline Aspect in degree & $\underset{\substack{\infty \\
-1}}{-1}$ & 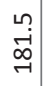 & 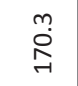 & $\underset{\substack{0\\
}}{\stackrel{0}{-1}}$ & 울 & 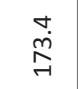 & 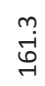 & $\underset{\stackrel{\sim}{r}}{\stackrel{-1}{\sim}}$ & $\begin{array}{l}0 \\
\dot{j} \\
\stackrel{-}{-1}\end{array}$ & $\begin{array}{l}\stackrel{+}{\oplus} \\
\stackrel{\leftrightarrow}{\leftrightarrow}\end{array}$ & $\stackrel{m}{m}$ & $\stackrel{\sim}{\dddot{n}}$ & $\underset{\substack{m \\
\infty \\
\sim}}{\sim}$ & $\underset{⿱}{\stackrel{+}{~}}$ & 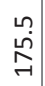 & $\underset{\substack{\sim \\
\sim}}{\sim}$ & $\stackrel{\stackrel{n}{n}}{\stackrel{n}{\digamma}}$ \\
\hline Slope in degree & $\stackrel{\Perp n}{\forall}$ & $\stackrel{0}{\stackrel{0}{r}}$ & $\stackrel{-}{\infty}+$ & $\stackrel{N}{r}$ & $\hat{\varphi}$ & $\hat{\infty}$ & $\stackrel{\infty}{0}$ & $\stackrel{\sim}{0}$ & $\hat{\sigma}$ & $\hat{\mathrm{n}}$ & $\stackrel{\sim}{\omega}$ & $\underset{\bullet}{\bullet}$ & $\stackrel{\infty}{\rightarrow}$ & $\ddot{n}$ & $\stackrel{-1}{r}$ & $\stackrel{0}{r}$ & $\underset{+}{\sigma}$ \\
\hline Elevation in $\mathrm{m}$ & ĩ & $\begin{array}{r}\hat{O} \\
\stackrel{d}{ \pm}\end{array}$ & $\begin{array}{l}\infty \\
\dot{\infty} \\
\stackrel{\sim}{\sim}\end{array}$ & 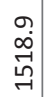 & ஸ̃ & 永 & $\stackrel{\stackrel{n}{n}}{\dddot{m}}$ & 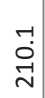 & $\begin{array}{l}0 \\
\infty \\
\infty \\
\infty \\
+\end{array}$ & 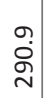 & ஸ̃ & 足 & 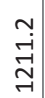 & $\stackrel{\infty}{\stackrel{\infty}{n}}$ & 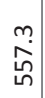 & 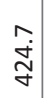 & 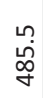 \\
\hline $\begin{array}{c}\text { Percentage of forest } \\
\text { cover gain }\end{array}$ & 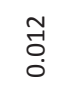 & \begin{tabular}{l}
8 \\
\hdashline \\
\hdashline
\end{tabular} & ঃ & $\begin{array}{l}8 \\
8 \\
\circ\end{array}$ & $\begin{array}{l}\stackrel{n}{O} \\
\stackrel{0}{0}\end{array}$ & ঃ & 웅 & $\stackrel{n}{\circ}$ & $\hat{o}$ & $\begin{array}{l}\hat{x} \\
0 \\
0\end{array}$ & $\stackrel{\circ}{\circ}$ & రั & $\begin{array}{l}\dot{0} \\
\dot{0} \\
0 \\
0\end{array}$ & $\underset{0}{\mathbb{1}}$ & $\begin{array}{l}n \\
\tilde{0} \\
0 \\
0\end{array}$ & $\begin{array}{l}\stackrel{0}{0} \\
0 \\
0 \\
0\end{array}$ & $\stackrel{\text { ñ }}{0}$ \\
\hline $\begin{array}{c}\text { Percentage of Forest } \\
\text { cover loss }\end{array}$ & $\begin{array}{l}0 \\
\infty \\
0 \\
0 \\
0\end{array}$ & \begin{tabular}{l}
8 \\
\hdashline \\
0
\end{tabular} & $\begin{array}{l}0 \\
\stackrel{-}{0} \\
0 \\
0\end{array}$ & 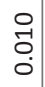 & $\begin{array}{l}-1 \\
\stackrel{-}{0} \\
0\end{array}$ & $\stackrel{\circ}{\circ}$ & $\begin{array}{l}8 \\
\circ \\
\circ\end{array}$ & 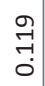 & $\underset{\dot{f}}{\grave{O}}$ & ન્ & 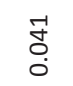 & ஜे & $\begin{array}{l}\ddot{\Xi} \\
\dot{0}\end{array}$ & 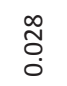 & $\begin{array}{l}\check{\Xi} \\
\dot{0}\end{array}$ & $\begin{array}{l}0 \\
\ddot{0} \\
0 \\
0\end{array}$ & 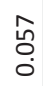 \\
\hline $\begin{array}{c}\text { Percentage of forest } \\
\text { cover }\end{array}$ & $\begin{array}{l}\text { ஜ } \\
\text { ஸึ่ }\end{array}$ & $\stackrel{+}{+}$ & นุ่ & $\begin{array}{l}\varphi \\
\dot{\sim} \\
\dot{\sim}\end{array}$ & i্d & $\begin{array}{l}\text { ํㅗㅇ } \\
\stackrel{0}{0}\end{array}$ & $\stackrel{8}{\circ}$ & $\stackrel{\infty}{i}$ & $\stackrel{n}{n}$ & $\underset{\infty}{\infty}$ & ヴ & กุ' & $\begin{array}{l}\dot{\varphi} \\
\dot{n}\end{array}$ & $\stackrel{\dot{m}}{m}$ & $\begin{array}{r}\dot{+} \\
\dot{0}\end{array}$ & กิ่ & $\begin{array}{l}\circ \\
\dot{\sigma}\end{array}$ \\
\hline $\begin{array}{l}\text { Species habitat area in } \\
\qquad \mathrm{Km}^{2}\end{array}$ & $\stackrel{\sim}{m}$ & 온 & $\underset{\substack{-m}}{\stackrel{m}{m}}$ & $\begin{array}{l}0 \\
\stackrel{0}{0} \\
\stackrel{y}{q}\end{array}$ & $\begin{array}{l}\infty \\
\dot{\varphi} \\
\stackrel{\mathfrak{N}}{\hat{O}} \\
\stackrel{+}{+}\end{array}$ & $\begin{array}{l}8 \\
\searrow \\
\stackrel{-}{-} \\
-1\end{array}$ & ঃ & $\begin{array}{l}\text { ठ } \\
\stackrel{0}{0} \\
\stackrel{-}{m}\end{array}$ & 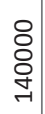 & ষ০ & ثें & ঃ & $\begin{array}{l}\stackrel{0}{0} \\
\stackrel{n}{6}\end{array}$ & 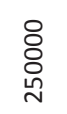 & 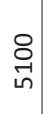 & 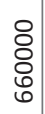 & 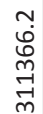 \\
\hline $\begin{array}{l}\text { Species number/species } \\
\text { scientific name }\end{array}$ & 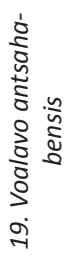 & 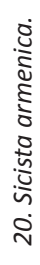 & 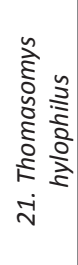 & 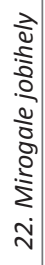 & 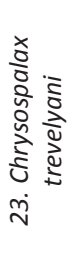 & 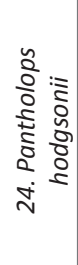 & 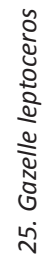 & 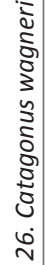 & 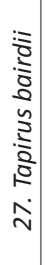 & 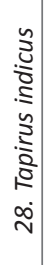 & 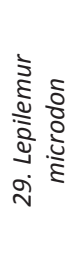 & 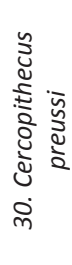 & 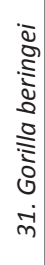 & 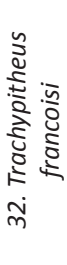 & 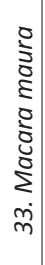 & 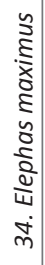 & $\begin{array}{l}0 \\
0 \\
0 \\
0 \\
0 \\
8 \\
5 \\
0 \\
0 \\
0 \\
0 \\
\text { U. }\end{array}$ \\
\hline
\end{tabular}


Table S4: Data source and variables used for GLM models

\begin{tabular}{|c|c|c|c|c|}
\hline $\begin{array}{c}\text { Variable data collection } \\
\text { for GLM }\end{array}$ & Variable name & Description & Data source & Variable calculation \\
\hline Survey/Observations & $\begin{array}{l}\text { GBIF species occur- } \\
\text { rences }\end{array}$ & $\begin{array}{l}\text { Species data records } \\
\text { were collected for } \\
\text { the period between } \\
2000 \text { and } 2015\end{array}$ & (GBIF.org 2018a) & - \\
\hline Survey/Observations & $\begin{array}{l}\text { GBIF species occur- } \\
\text { rences }\end{array}$ & $\begin{array}{l}\text { Species occurrence } \\
\text { records between } \\
2000 \text { and } 2017\end{array}$ & (GBIF.org 2018b) & - \\
\hline $\begin{array}{c}\text { Time (year of forest data } \\
\text { observations) }\end{array}$ & $\begin{array}{l}\text { Forest cover } 2000 \text {; } \\
\text { forest cover change } \\
2000-2014\end{array}$ & $\begin{array}{l}\text { LANDSAT images } \\
\text { data calculation }\end{array}$ & (Hansen et al., 2013) & $\begin{array}{l}\text { Percentage per output grid cell } \\
\text { of approximately } 900 \mathrm{~m} 2\end{array}$ \\
\hline See species occurrences & $\begin{array}{l}\text { Species pseudo-ab- } \\
\text { sences (see species } \\
\text { occurrences) }\end{array}$ & $\begin{array}{l}\text { A randomly selected } \\
\text { species pseudo-ab- } \\
\text { sences }\end{array}$ & $\begin{array}{l}\text { Species habitat areas } \\
\text { of IUCN (IUCN 2016) }\end{array}$ & $\begin{array}{c}\text { Randomly selected } 30 \text { species } \\
\text { pseudo-absences outside and } \\
\text { around of a species habitat } \\
\text { area(s) }\end{array}$ \\
\hline Expert contribution & Species occurrences & $\begin{array}{l}\text { A randomly selected } \\
\text { species occurrences }\end{array}$ & $\begin{array}{l}\text { Species habitat areas } \\
\text { of IUCN (IUCN 2016) }\end{array}$ & $\begin{array}{c}30 \text { species occurrences using } \\
\text { Create Random Points in } \\
\text { ArcGIS } 10.3 \text { within a species } \\
\text { habitat area(s) }\end{array}$ \\
\hline Expert contribution & $\begin{array}{c}\text { Terrestrial Protected } \\
\text { Areas }\end{array}$ & $\begin{array}{l}\text { Point data of the } \\
\text { version July } 2015 \\
\text { of the }\end{array}$ & $\begin{array}{l}\text { World Database } \\
\text { on Protected areas } \\
\text { (IUCN and UNEP- } \\
\text {-WCMC 2015) }\end{array}$ & $\begin{array}{l}\text { Straight line in Spatial analyst } \\
\text { in ArcGIS } 10.3\end{array}$ \\
\hline & $\begin{array}{l}\text { Terrain Ruggedness } \\
\text { Index (TRI) }\end{array}$ & $\begin{array}{l}\text { Derived from ele- } \\
\text { vation }\end{array}$ & See elevation & $\begin{array}{c}\text { Morphometry of SAGA GIS } \\
6.1 .0\end{array}$ \\
\hline & $\begin{array}{l}\text { Slope of the terrain } \\
\text { (see elevation) }\end{array}$ & $\begin{array}{l}\text { Derived from eleva- } \\
\text { tion, 1-minute arc } \\
\text { raster data }\end{array}$ & See elevation & $\begin{array}{l}\text { Slope in Spatial Analyst in } \\
\text { ArcGIS } 10.3\end{array}$ \\
\hline & $\begin{array}{l}\text { Aspect of the terrain } \\
\text { (see elevation) }\end{array}$ & $\begin{array}{l}\text { Derived from ele- } \\
\text { vation 1-minute arc } \\
\text { raster data }\end{array}$ & See elevation & $\begin{array}{l}\text { Aspect in Spatial Analyst in } \\
\text { ArcGIS } 10.3\end{array}$ \\
\hline \multirow[t]{3}{*}{ Survey } & $\begin{array}{c}\text { Elevation above sea } \\
\text { level }\end{array}$ & $\begin{array}{c}\text { 1-minute arc raster } \\
\text { data }\end{array}$ & (IUCN 2017) & \\
\hline & Diversity & $\begin{array}{l}\text { Derived from forest } \\
\text { cover of the year } \\
2000\end{array}$ & See forest cover & $\begin{array}{c}\text { Pattern Analysis of SAGA GIS } \\
6.1 .0\end{array}$ \\
\hline & Fragmentation & $\begin{array}{l}\text { Derived from forest } \\
\text { cover of the year } \\
2000\end{array}$ & See forest cover & $\begin{array}{c}\text { Pattern Analysis of SAGA GIS } \\
6.1 .0\end{array}$ \\
\hline \multirow[t]{2}{*}{ Survey } & Pastureland & $\begin{array}{l}\text { Raster grid data with } \\
\text { a resolution of } 5 \mathrm{~km} \\
\quad \times 5 \mathrm{~km}\end{array}$ & $\begin{array}{l}\text { Data of the year } 2010 \\
\text { (Ramankutty et al., } \\
\text { 2010) }\end{array}$ & $\begin{array}{c}\text { The percent of pixel area of } \\
\text { pastureland }\end{array}$ \\
\hline & Cropland & $\begin{array}{l}\text { Raster grid data with } \\
\text { a resolution of } 5 \mathrm{~km} \\
\quad \times 5 \mathrm{~km}\end{array}$ & $\begin{array}{l}\text { Data of the year } 2010 \\
\text { (Ramankutty et al., } \\
\text { 2010) }\end{array}$ & $\begin{array}{l}\text { The percent of pixel area of } \\
\text { cropland }\end{array}$ \\
\hline
\end{tabular}




\section{S5. Hypothesis on natural factors}

Environmental variables were related to food abundance and refuge areas of mammals. Natural factors consisted of raster layers of forest cover, forest cover change, elevation, slope, aspect, pastures, distance variable of protected forests, Terrain Ruggedness Index and landscape metrics of forest patch diversity and fragmentation. Large-carnivore species like lynx (Lynx pardinus), tigers (Panthera tigris) need stable and undisturbed dense forests as well as elevated land and topography that provide suitable habitats for food and refuge and breed (Fernández et al., 2003; Kanagaraj et al., 2011). All 35 mammal terrestrial species were assumed to rely on forests as forests were the first preferable habitat for them followed by shrub lands and grasslands (IUCN 2016). The changes in forest cover (e.g., from forests to cropland) could be a threat to large-body mammal species as Catopuma badia (Mohd-Azlan \& Sanderson 2007). Pekin and Pijanowski (2012) found that disturbed and overused forests may threat mammal species at a global scale. Small mammals tend to use forests, for example, in Brazilian Atlantic forests, surrounded by pastures and tree crops (Estavillo et al., 2013) and primates were located in the protected areas with a forest cover above 50 percent (Nüchel et al., 2018). Small mammals can use farmland in some areas (see, e.g., Rogers \& Gorman 1995). Low-intensity cropland and rangelands (pastures) may help keep up the biodiversity of mammals, while high-intensity cropland can significantly threat mammal species at global level (Pekin \& Pijanowski 2012). Generally, cropland could be a suitable area for food to small mammal species, but not necessarily to large-body carnivore mammals. I assumed that low intensity agriculture and pastureland in tropical forested areas could be used as refuge (shelter) (Ester et al., 2018) areas for endangered mammal species.

A set of variables was assumed to plausibly explain the existence of all these endangered species in the wild. Environmental variables (elevation, slope, aspect), protected or non-protected forests were commonly often used in species modelling (Fernández et al., 2003; Gaillard et al., 2010; Kanagaraj et al., 2011; Soto \& Palomares, 2015; Nüchel et al., 2018). These environmental variables explained mostly the existence of species in their habitat areas for food and or refuge. Forests were the most preferred habitats for terrestrial mammal species (IUCN 2016) including 35 endangered mammal species.
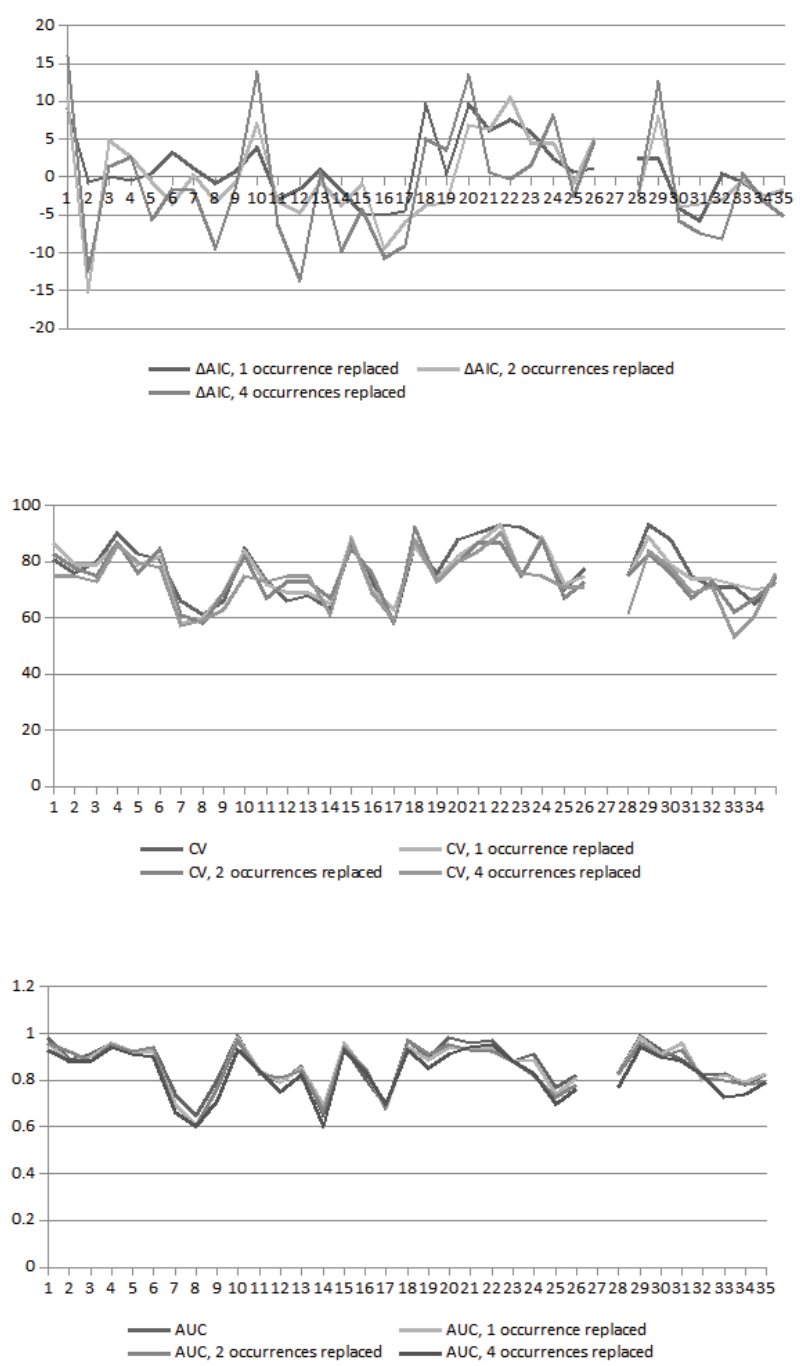

Figure S6: GLM results obtained by randomly replacing one, two and four occurrences with no occurrences for endangered mammal species (indicated by number species from one to thirty-five) models: (a) the change of Akaike Information Criteria (AIC), (b) the ten-fold cross-validation (CV) and (c) the Receiver Operating Characteristic Curve (AUC). There were no GLM model results for endangered mammal species of Tapirus bairdii numbered twenty-seven, because there were no variables resulting from Kruskal-Wallis test. 